\title{
On couple-stress elasto-plastic constitutive frameworks for glassy polymers
}

\author{
Farid Alisafaei, Chung-Souk Han, Nitin Garg \\ University of Wyoming, Department of Mechanical Engineering, 1000 E. University Ave., Laramie, WY 82071, USA
}

\begin{abstract}
Various experimental studies have revealed length scale dependent deformation of different materials at micron and sub-micron length scales. In metals, such length scale dependent deformations are considered to be associated with geometrically necessary dislocations arising from an increase in strain gradients related to non-uniform plastic deformation. Recent experiments, however, have shown that the nature of length scale dependent deformation in polymers is quite different from metals as in polymers size effects have been observed in pure elastic as well as in elasto-plastic deformation. It is shown in this study that similar to metals, size effects in polymers can be associated with an increase in higher order displacement gradients with decreasing length scale. However, the experimental results discussed here reveals that, in contrast to metals, these higher order displacement gradients are mainly of elastic nature even when glassy polymers deform elasto-plastically. Taking the effects of these elastic higher order displacement gradients into account, a couple-stress theory is developed to predict size effect in glassy polymers with elasto-plastic deformation.
\end{abstract}

Keywords: Length scale dependent deformation; Glassy polymers; Couple-stress; Elasto-plasticity

\section{Introduction}

Polymeric materials at small dimensions have found widespread technical applications in sensors, optical/electronic devices, etc. Subsequently, a great effort has been directed toward the understanding of the deformation mechanisms of polymers at nano- to micrometer length scales where the deformation characteristics of some polymers can be very different in comparison to bulk deformation behavior. As in metals (Nix \& Gao, 1998; Ma \& Clarke, 1995; Fleck et al., 1994; Liu et al., 2013; Swadener et al., 2002; Motz et al., 2005), length scale dependent behavior has been also experimentally observed in polymers (Han, 2010; Alisafaei \& Han, 2015) at micron to submicron length scales by beam bending testing (Lam et al., 2003; McFarland \& Colton, 2005), nanoindentation (Briscoe et al., 1998; Chong \& Lam, 1999; Alisafaei et al., 2013; 2014) and bending test of nanofibers (Sun et al., 2008; Arinstein et al., 2007). Various polymers exhibited such length scale dependent deformation including epoxy (Chong \& Lam, 1999; Lam \& Chong, 1999; 2000; 2001; Lam et al., 2003; Alisafaei et al., 2014; Dutta et al., 2004; Sanchez et al., 2011), polycarbonate (PC) (Briscoe et al., 1998; Chong \& Lam, 1999; Balta Calleja et al., 2004), polydimethylsiloxane (PDMS) (Charitidis, 2011; Alisafaei et al., 2013; Wrucke et al., 2013; Han et al., 2015; Lim \& Chaudhri, 2006; Koumoulos et al., 2014), polystyrene (PS) (Briscoe et al., 1998; Balta Calleja et al., 2004; Tjernlund et al., 2004), silicone rubber/elastomer (Zhang \& Xu, 2002; Xu et al., 2005;

- Corresponding author. Postal address: University of Wyoming, Department of Mechanical Engineering, Dept. 3295,1000 E. University Avenue, Laramie, WY 82071, USA. Tel.: +1 307766 2930, E-mail: chan1@uwyo.edu 
Tatiraju \& Han, 2010; Chandrashekar et al., 2015), polymethyl methacrylate (PMMA) (Briscoe et al., 1998; Balta Calleja et al., 2004), Nylon 66 (PA66) (Shen et al., 2005), Nylon 11 (PA11) (Hu et al., 2006), polypropylene (PP) (McFarland \& Colton, 2005), polyimide (PI) (Lu et al., 2010), polyamide (PA) (Seltzer et al., 2011), polyvinyl chloride (PVC) (Balta Calleja et al., 2004) and polystyrene acrylonitrile (SAN) (Balta Calleja et al., 2004). In addition to the above mentioned polymers, size effects have been also reported in elastic deformation of foams and circular cell polycarbonate honeycomb where a Cosserat elastic continuum model and micropolar elasticity were applied to describe their length scale dependent deformations (Lakes, 1986, 1991; Mora \& Waas, 2002; 2007).

While classical elasto-plasticity theory is not capable of predicting the length scale dependent behavior at nano-micro length scales, there are only few studies on the origin and modeling of length scale dependent elasto-plastic deformation in polymers. Based on the molecular theory of yielding in glassy polymers and a molecular kinking mechanism, a strain gradient plasticity law was developed in (Lam \& Chong, 1999) where a strain gradient plasticity parameter dependent on molecular and temperature was proposed and related to indentation hardness. The developed depth dependent indentation hardness model was used in (Chong \& Lam, 1999) to predict indentation size effects of the thermoset epoxy and thermoplastic polycarbonate. Strain gradient plasticity effect presented in (Lam \& Chong, 1999) was incorporated into viscoelastic-plastic constitutive model of Govaert et al. (2000) to capture length scale dependent deformation of glassy polymers observed in nanoindentation tests (Swaddiwudhipong et al., 2005). Recently, extending the mechanism based theories of crystalline metals, a strain gradient plasticity formulation was developed in (Voyiadjis et al., 2014) for semi-crystalline polymers where length scale dependent deformation of semi-crystalline polymers was attributed to crystalline sub-phase embedded within an amorphous matrix. Two kink mechanisms of Grounded Kink and Active Kink were utilized to respectively capture non-localized effects (e.g. uniform plastic deformation) and incompatible viscoplastic deformation effects induced by non-uniform slippage mechanisms (e.g. localized plastic deformation around the indenter tip in indentation tests). Furthermore, using Cosserat continuum formulation, length scale dependent deformation of amorphous polymers was modeled in (Voyiadjis et al., 2014) where a flow rule was proposed for this kind of polymers. In addition to the above mentioned strain gradient plasticity continuum models, a molecular dynamics based approach was presented in (Ghosh et al., 2013) for non-local elastic modeling of epoxy using an atomistically-informed kernel.

As presented in the above mentioned strain gradient plasticity models (Lam \& Chong, 1999; Swaddiwudhipong et al., 2005), the deformation mechanisms of polymers at nano- to micrometer length scales can be described with strains and their gradients. Similar to size effect in metals, these strain gradients and subsequently size effect in polymers are usually considered to be of plastic nature (Lam \& Chong, 1999; Swaddiwudhipong et al., 2005). However, experiments discussed in the following section illustrate that size effect characteristics in polymers are quite different from metals which would motivate a different approach to model length scale dependent deformation mechanisms in polymers. To capture these experimental findings, a couple-stress formulation is proposed for length scale dependent elastoplastic deformation in glassy polymers at micron to submicron length scales. In Section 2, experimental data of length scale dependent deformation in glassy polymers is compared with corresponding experiments of metals to illustrate the different characteristics. To describe such deformation behavior, an elasto-plastic couple-stress formulation is developed in Section 3 for glassy polymers at micron to submicron length scales. The couple-stress formulation is discussed in Section 4 followed by conclusion remarks in Section 5. 


\section{Length scale dependent deformation characteristics in polymers}

Based on experimental data, the characteristics of length scale dependent deformation in polymers are discussed in this section that will be applied as the basis for length scale dependent model proposed in Section 3. While the mechanical response of polymeric materials are clearly known to be time dependent (Oyen \& Cook, 2003; Zhai \& McKenna, 2014; Anand \& Ames, 2006; Gudmundson, 2006; Sperling, 1993), for simplicity we restrict our attention to length scale dependent elasto-plastic deformation in glassy polymers under conditions where rate effects are assumed to be negligible.

\subsection{Size effects in metals and polymers}

In metals, length scale dependent deformation at micron to submicron length scales is commonly associated with the notion of geometrically necessary dislocations that can be in turn related to strain gradients of non-uniform plastic deformation (Fleck et al., 1994; Nix \& Gao, 1998). Length scale dependent deformation has been also observed in polymers at micron to submicron length scales (Alisafaei \& Han, 2015). In a difference to metals, however, size effects in polymers are also observed in elastic deformation, as for instance in (Lam et al., 2003), micro-beam bending experiments of epoxy with elastic deformation revealed remarkable increase in the (normalized) bending stiffness with decreasing beam thicknesses, see Fig. 1(a). As it can be seen in Fig. 1 (a), based on the classical (local) elasticity beam theory, the bending stiffness is independent of beam thickness for a fixed $a / h$ ratio. However, as depicted in Fig. 1 (a), the experimental results of (Lam et al., 2003) show that for a fixed $a / h$ ratio the bending stiffness significantly increases with decreasing beam thickness from 115 to $20 \mu \mathrm{m}$.

The load-deflection data shown in Fig. 1(b) illustrates the reversible elastic behavior of epoxy microbeams revealing that, in contrast to metals, size effects in polymers may not be related only to plastic deformation. Similar stiffening has been observed in micro-beam bending experiments of polypropylene (McFarland \& Colton, 2005) where elastic stiffness of polypropylene significantly increases with decreasing thickness. These beam bending experiments would suggest that size effects in polymers may be of elastic nature even when polymers deform elasto-plastically.

To model such behavior, the couple stress elasticity theory of Yang et al. (2002) has been quite successful for which a numerical approach has been suggested in (Garg \& Han, 2013). Applying this numerical approach with $E=1.5 \mathrm{GPa}$ (elastic modulus of epoxy) and $v=0.355$ (Poisson's ratio of epoxy) as in (Lam et al., 2003), and $\widetilde{K}=0.116 \mathrm{~N}$ (non-local material constant $\widetilde{K}$ which is related to the length scale parameter $\ell$ in (Yang et al., 2002) by $\widetilde{K}=3 G \ell^{2}$ with $G$ being the shear modulus of epoxy), very good agreement between the experiments and numerical simulations is found, see Fig. 1(a). Eight node quadrilateral elements with selective reduced integration (using four gauss points) are applied in a mesh with 640 elements ( 8 by 80 ) where meshes with higher number of elements did not noticeably change the simulation results (see (Garg \& Han, 2013) for more details).

To examine whether the size effect in polymers are of elastic nature and to shed light on its origin, nanoindentation tests are carried out on epoxy as a glassy polymer. The results obtained from these tests are analyzed and compared with the ones reported in the literature for metals to study the difference between nature of size effect in metals and polymers. 


\subsection{Nanoindentation of metals and polymers}

Hardness. Applying Oliver \& Pharr's (1992) approach with the Berkovich tip, in Fig. 2 the indentation hardness, $H_{\mathrm{I}}=F / A_{\mathrm{c}}$ (where $F$ is the applied force and $A_{\mathrm{c}}$ is the contact area, see Oliver \& Pharr (1992) or ISO14577-1, 2002), is shown over a broad range of the indentation depths for aluminum (data redrawn from Saha \& Nix, 2002; Rodriguez \& Gutierrez, 2003). Therein, an indentation size effect can be clearly seen as the hardness increases significantly with decreasing indentation depth. To study the indentation size effect in glassy polymers, similar nanoindentation tests have been carried out on epoxy at different indentation depths ranging from 3000 to $30 \mathrm{~nm}$. The epoxy samples were prepared by mixing commercially available liquid epoxy resin, EPON 828 (bisphenol A-epichlorohydrin), as base agent with $20 \mathrm{phr}$ (parts per hundred resin) curing agent EPIKURE 3223, diethylenetriamine (DETA). More details on the sample preparations can be found in (Alisafaei et al., 2014). All indentation tests were performed with the Agilent G200 Nano Indenter system (Agilent Technologies, Oak Ridge, TN, USA) in force controlled mode with linear force-time relations for both loading and unloading. To compare with the aluminum hardness data of (Saha \& Nix, 2002; Rodriguez \& Gutierrez, 2003), indentation tests on epoxy were performed with a sharp Berkovich tip with a curvature radius of less than $20 \mathrm{~nm}$ according to the manufacturer's specifications. The bluntness of the Berkovich tip was found to be slightly higher than the specified $20 \mathrm{~nm}$ as it was measured to be $24.4 \mathrm{~nm}$ and $40.2 \mathrm{~nm}$ by two different approaches (see Alisafaei et al., 2014) which may indicate a slight blunting of the tip due to use on harder materials like fused silica. All Berkovich indentations were conducted at $23{ }^{\circ} \mathrm{C}$ using a Dynamic Contact Modulus (DCM) indenter head with $20 \mathrm{~s}$ loading, holding and unloading times. To detect initial contact of the tip with the sample surface, a surface detection criterion of $50 \mathrm{~N} / \mathrm{m}$ was applied; see (Alisafaei et al., 2013) for more details. The minimum distance between two individual indents was $50 \mu \mathrm{m}$. Fig. 3 depicts the indentation hardness of epoxy obtained by the same method of Oliver \& Pharr (O\&P) using the above mentioned Berkovich tip. As in Fig. 2, size effects are also observed in Fig. 3 where $H_{\mathrm{I}}$ of epoxy increases with decreasing indentation depth. It can be seen that $H_{\mathrm{I}}$ obtained from our experiments is in good agreement with an earlier study of Lam \& Chong (2000) on an epoxy with the same $20 \%$ hardener. In addition to the indentation hardness, $H_{\mathrm{I}}$, the universal hardness, $H_{\mathrm{U}}=F / A_{\mathrm{n}}$ (where $A_{\mathrm{n}}$ is the nominal area below the initial surface as defined in ISO14577-1, 2002), of epoxy is also plotted in Fig. 4 illustrating that both $H_{\mathrm{I}}$ and $H_{\mathrm{U}}$ exhibit similar factors of increases with decreasing indentation depth (the universal hardness is applicable to both elastic and inelastic materials, see (ISO14577-1, 2002)).

As the hardness values involve both elastic and plastic deformation/resistance, from the hardness values it cannot be distinguished whether the indentation size effect is of elastic or plastic nature (or the combination of both). To understand the underlying nature of size effects in polymers, we need to analyze a material property which only contains either elastic or plastic resistance (not both of them together). To this end, the indentation elastic moduli of epoxy and aluminum both obtained by Oliver \& Pharr (1992) method will be studied in the following. Note that the elastic modulus obtained according to Oliver \& Pharr (1992) is only related to the elastic resistance at unloading and can be therefore useful for understanding of the rheological differences between the size effects in polymers and metals.

Elastic modulus. Following Oliver \& Pharr (1992) and using a Berkovich tip, the elastic modulus, $E_{\mathrm{B}}^{\mathrm{O} \& \mathrm{P}}$, is defined via

$$
\frac{1}{E_{\mathrm{r}}}=\frac{1-v^{2}}{E_{\mathrm{B}}^{\mathrm{O} \& \mathrm{P}}}+\frac{1-v_{\mathrm{i}}^{2}}{E_{\mathrm{i}}},
$$


where $v$ and $v_{\mathrm{i}}$ are respectively the Poisson's ratios of sample and indenter tip, $E_{\mathrm{i}}$ is the elastic modulus of the tip, and $E_{\mathrm{r}}$ is the reduced elastic modulus which is obtained by

$E_{\mathrm{r}}=\frac{\sqrt{\pi}}{2} \frac{S}{\sqrt{A}}$,

in which $S$ in the initial slope of unloading curve (see Fig. 5), $A$ is the projected contact area of indentation at the contact depth $h_{\mathrm{c}}$ (see Oliver \& Pharr, 1992, for more details). From Eqs. (1) and (2), it follows that $E_{\mathrm{B}}^{\mathrm{O} \& \mathrm{P}}$ is approximately proportional to $S / \sqrt{A}$ or $S / h_{\mathrm{c}}\left(\right.$ Oliver \& Pharr, 1992) for $E_{\mathrm{i}} \gg E_{\mathrm{B}}^{\mathrm{O} \& \mathrm{P}}$. Note that most polymers are quite soft compared to diamond tips $\left(E_{\mathrm{i}} \gg E_{\mathrm{B}}^{\mathrm{O} \& \mathrm{P}}\right)$ so that the second term in the right hand of Eq. (1) can be assumed to be zero, $E_{\mathrm{B}}^{\mathrm{O} \& \mathrm{P}}=E_{\mathrm{r}}\left(1-v^{2}\right)$, yielding $E_{\mathrm{B}}^{\mathrm{O} \& \mathrm{P}} \propto S / \sqrt{A}$. As mentioned above, the material stiffness $S$ is determined from the initial slope of the unloading curve and is therefore not associated with plastic deformation.* As the slope of unloading curve $S$ involves only elastic deformation (elastic unloading), the parameter $S / \sqrt{A}$ (with a unit of $\mathrm{N} / \mathrm{m}^{2}=\mathrm{Pa}$ ) can be considered as an elastic resistance if viscous effects are assumed to be negligible.

The elastic modulus, $E_{\mathrm{B}}^{\mathrm{O} \& \mathrm{P}}$, of aluminum obtained from the same experiments of Fig. 2 is depicted in Fig. 6. It can be seen in Fig. 6 that $E_{\mathrm{B}}^{\mathrm{O} \& \mathrm{P}}$ of aluminum redrawn from (Saha \& Nix, 2002; Rodriguez \& Gutierrez, 2003) stays constant with indentation depth. This would imply that the material is homogeneous through the depth. However, in contrast to Fig. 6, the elastic modulus of epoxy determined by Oliver \& Pharr (1992) method does not remain constant with indentation depth as it is shown in Fig. 7 where $E_{\mathrm{B}}^{\mathrm{O} \& \mathrm{P}}$ of epoxy increases with decreasing indentation depth. Similar increase in the elastic modulus with decreasing depth were also observed in (Dutta et al., 2004; Sanchez et al., 2011) where both indentation hardness and elastic modulus of epoxy obtained by a Berkovich tip increased with decreasing indentation depth. Note that while the elastic moduli of both aluminum and epoxy have been obtained by the same method of Oliver \& Pharr (1992), in contrast to aluminum, $E_{\mathrm{B}}^{\mathrm{O} \& \mathrm{P}}$ of epoxy increases with decreasing indentation depth.

A possible explanation for this increase of $E_{\mathrm{B}}^{\mathrm{O} \& \mathrm{P}}$ in epoxy would be a change in the material properties with depth. To examine this, the elastic modulus of epoxy is determined by a different approach applying a spherical tip with a radius of $250 \mu \mathrm{m}$ (Alisafaei et al., 2014). All tests with the spherical tip were performed using the same indenter system in force controlled mode with linear force-time relations for both loading and unloading. These tests with the spherical tip were carried out at the same temperature $23{ }^{\circ} \mathrm{C}$ using an XP indenter head with $20 \mathrm{~s}$ loading and unloading times (no holding time) where a surface detection criterion of $200 \mathrm{~N} / \mathrm{m}$ was applied (see Alisafaei et al., 2013). It can be seen in Fig. 7 that the both elastic moduli $E_{\text {sphere }}^{\text {Hertz }}$ (determined by the spherical tip and Hertz (1882) theory) and $E_{\text {sphere }}^{\text {O\&P }}$ (determined with a sphere and Oliver \& Pharr (1992) method) remain almost constant in the whole range of 3700 to $400 \mathrm{~nm}$. These results would indicate that the increase of $E_{\mathrm{B}}^{\mathrm{O} \& \mathrm{P}}$ in epoxy (with decreasing depth) obtained by the Berkovich tip cannot be attributed to changes in the material properties of epoxy with depth. The results obtained from the spherical tests, i.e. $E_{\text {sphere }}^{\text {Hertz }}$ and $E_{\text {sphere }}^{\text {O\&P }}$ depicted in Fig. 7 would indicate that similar to the results shown in Fig. 6 for aluminum, the elastic modulus of epoxy is also constant through thickness.

\footnotetext{
* Assuming that the behavior of material is time independent for simplicity, the total deformation of the material can be decomposed into elastic and plastic deformations where unloading section only contains the elastic deformation.
} 
To study the differences between $E_{\mathrm{B}}^{\mathrm{O} \& \mathrm{P}}$ obtained with a Berkovich tip on one side and $E_{\text {sphere }}^{\mathrm{Hertz}}$ and $E_{\text {sphere }}^{\text {O\&P }}$ obtained with a spherical tip on the other side over the indentation depths, a more detailed look is pursued. More specifically, it is here argued that the increase of $E_{\mathrm{B}}^{\mathrm{O} \& \mathrm{P}}$ in epoxy with decreasing indentation depth is related to different rheological characteristics in the size effects of polymers compared to metals which will be examined in the following.

The elastic resistance $S / \sqrt{A}$ in Eq. (1) is constant with $h_{\max }$ (maximum indentation depth shown in Fig. 5) for aluminum as $E_{\mathrm{B}}^{\mathrm{O} \& \mathrm{P}}$ of aluminum does not change with depth shown in Fig. 6. Length scale dependent deformation in metals is associated with plastic deformation and therefore no length scale dependent stiffening is expected during unloading (which is only associated with elastic deformation). Correspondingly, the elastic resistance $S / \sqrt{A}$ does not increase with decreasing $h_{\max }$ in aluminum, see Fig. 6. In epoxy, however, the elastic resistance $S / \sqrt{A}$ increases with decreasing $h_{\max }$ as $E_{\mathrm{B}}^{\mathrm{O} \& \mathrm{P}}$ of epoxy depicted in Fig. 7 increases with decreasing indentation depth. This increase in the elastic resistance of epoxy with decreasing $h_{\max }$ would indicate that size effects in epoxy, in contrast to metals, are significantly related to elastic deformation which would be in agreement with the micro-beam bending experiments of Lam et al. (2003) and McFarland \& Colton (2005) where size effects are also associated with elastic deformation.

Concerning the significance of the elastic nature in the length scale dependent deformation of epoxy, one may compare the increases in $E_{\mathrm{B}}^{0 \& \mathrm{P}}$ and $H_{\mathrm{I}}$ with decreasing $h_{\max }$. Evaluation of the data in Figs. 3, 4 and 7 shows that the increase of $E_{\mathrm{B}}^{\mathrm{O} \& \mathrm{P}}$ is approximately proportional to the increase of $H_{\mathrm{I}}$ with decreasing $h_{\max }$. This may indicate that there is almost the same increase in the elastic resistance (during unloading) and elasto-plastic resistance (during loading) which would suggest that size effect in polymers is significantly associated with elastic deformation. As will be seen below, such an increase in both $E_{\mathrm{B}}^{O \& P}$ and hardness of epoxy with decreasing depth, shown above and in (Alisafaei et al., 2014; Dutta et al., 2004; Sanchez et al., 2011), have been also observed in other polymers such as PMMA, PS and PC (Briscoe et al., 1998; Balta Calleja et al., 2004). This would suggest that length scale dependent deformation in polymers has at least a strong elastic rheological nature which in turn would suggest that length scale dependent deformation in polymers is significantly related to elastic deformation even when they deform elasto-plastically.

Higher order displacement gradients. Presuming that length scale dependent deformation in polymers is associated with elastic deformation, then the elastic modulus obtained with the O\&P method (which is based on the analysis of unloading curve) will increase with decreasing $h_{\max }$ when a Berkovich tip is applied. However, it can be seen in Fig. 6 that, in contrast to $E_{\mathrm{B}}^{\mathrm{O} \& \mathrm{P}}$ of Berkovich tip, the elastic moduli of epoxy determined by the spherical tip, $E_{\text {sphere }}^{\mathrm{Hertz}}$ and $E_{\text {sphere }}^{\mathrm{O} \text {, } \mathrm{P}}$ remain more or less constant with $h_{\max }$ indicating that the elastic resistance of epoxy does not increase with decreasing $h_{\text {max }}$ when a spherical tip is applied. This raises the question why elastic resistance increases with decreasing $h_{\text {max }}$ when a sharp Berkovich tip is applied.

As mentioned above, size dependent deformation of epoxy is observed to be dependent on the geometry of indenter tip. The same trend is observed in metals where indentation depth dependent deformation of metals has been found to be tip geometry dependent (Swandener et al., 2002). Similar to the present findings for glassy polymers, the hardness of annealed iridium obtained by a sharp Berkovich tip is found to increase with decreasing $h_{\max }$ in (Swandener et al., 2002) whereas the hardness is reported to be $h_{\max }$ independent when a spherical tip is applied. 
In metals, size dependent deformation is usually associated with geometrically necessary dislocation densities (Dahlberg et al., 2014; Lyu et al., 2015) arising from increasing strain gradients with decreasing length scale (see also Taheri-Nassaj \& Zbib, (2015) for stress gradient plasticity model). Although the notion of geometrically necessary dislocations cannot be applied to polymers, similar to metals, increases of strain/rotation gradients or generally second order displacement gradients (with decreasing length scale) are suggested to describe and predict size effects in polymers (Han \& Nikolov, 2007; Nikolov et al., 2007; Chong \& Lam, 1999; Lam \& Chong, 1999, 2000, 2001; Lam et al., 2003; Voyiadjis et al., 2014; Swaddiwudhipong et al., 2005). From a mechanistic point of view, the increases in rotation gradients (with decreasing length scale) can be rationalized with the finite bending stiffness of polymer chains and their interactions (Han \& Nikolov, 2007; Nikolov et al., 2007) which is discussed below.

The relation between second order displacement gradients (which will be denoted with $\chi$ in the following) and tip geometry in indentation test is illustrated in Fig. 8 where the change of these higher order gradients $\chi$ with $h_{\max }$ for different tip geometries is shown. As seen therein, the higher displacement gradients increase with decreasing $h_{\max }$ for the Berkovich tip. However, for a spherical tip, the higher displacement gradients should be mostly inversely proportional to the tip radius and essentially independent of indentation depth (Tymiak, et al., 2001; Gerberich et al., 2002; Swadener et al., 2002). The different trends in the determined elastic moduli of epoxy with $h_{\max }$ shown in Fig. 7 can be therefore described with second order displacement gradients $\chi$ as they increase with decreasing depth for a Berkovich tip while they remain constant ( $\chi$ is essentially independent of $h_{\max }$ ) when a spherical tip is applied. Considering that length scale dependent deformation is, at least to a good portion, of elastic nature in glassy polymers (as discussed above), it would correspondingly follow that $\chi$ is also, at least to a significant part, reversible.

Although such a conclusion is mostly based on the experiments of the thermosetting epoxy shown in Figs. 3, 4 and 7, similar trends are also present in thermoplastic polymers in the glassy state. In Figs. 911 , for instance, the normalized values of elastic modulus and hardness of three thermoplastic polymers PS, PC and PMMA are plotted where a Berkovich tip was applied. The elastic modulus and hardness data of these three thermoplastic polymers obtained from nanoindentation experiments of (Briscoe et al., 1998; Balta Calleja et al., 2004) are normalized by their bulk values (their elastic modulus and hardness at large indentation depths). It is seen in Figs. 9-11 that for all three thermoplastic polymers PS, PC and PMMA, both elastic modulus and hardness (indentation and universal hardness) significantly increase with decreasing $h_{\max }$. Such increases in elastic modulus (representing elastic resistance) and hardness (representing elasto-plastic resistance) with decreasing $h_{\max }$ illustrates that the length scale dependent deformation in thermoplastic polymers is also at least for a significant part elastic.

\section{Couple-stress elasto-plasticity theory}

The length scale dependent elasto-plastic deformation in metals at micron to submicron scales is often modeled with terms related to the gradients of the plastic strain tensor (see, e.g., Arsenlis \& Parks, 1999; Fleck et al., 1994; Huang et al., 2004; Han et al., 2007). As length scale dependent deformations in the considered glassy polymers are mainly of elastic nature, a different approach should be pursued in polymers. Although rate dependent deformation is much more pronounced in polymers (see, e.g., Sperling, 1993; Zhai \& McKenna, 2014; Krairi \& Doghri, 2014) than in metals, the rate effects for the following material description is neglected assuming that during the deformation process the deformation 
rates remain within a similar range (changes in the deformation due to rate differences are negligible). Furthermore, we assume that the considered polymers exhibit isotropic deformation behavior.

To account for the effect of strain/rotation gradients on the deformation mechanism of polymers, the total deformation energy density $W$ is augmented by a deformation work density $W_{\chi}$ (related to rotation gradients) as follows

$W=W_{\varepsilon}^{\mathrm{e}}+W_{\varepsilon}^{\mathrm{p}}+W_{\chi}$,

where the local terms $W_{\varepsilon}^{\mathrm{e}}$ and $W_{\varepsilon}^{\mathrm{p}}$ are respectively the elastic and plastic work densities (the subscript $\varepsilon$ indicates the local nature of these components). The non-local term $W_{\chi}$, which is the rotation gradients contribution to the total energy density $W$, has significant elastic rheological characteristics in glassy polymers and is expressed as a function of rotation gradients (Han \& Nikolov, 2007; Nikolov et al., 2007). Similar to the local terms $W_{\varepsilon}^{\mathrm{e}}$ and $W_{\varepsilon}^{\mathrm{p}}$, a corresponding decomposition of the non-local term $W_{\chi}$ would be possible, i.e.

$W_{\chi}=W_{\chi}^{\mathrm{e}}+W_{\chi}^{\mathrm{p}}$

where the elastic component $W_{\chi}^{\mathrm{e}}$ should not be neglected as, following the arguments of Section 2, the length scale dependent deformation of the considered glassy polymers is, at least to a strong part, of elastic nature. Correspondingly, the simplest assumption in view of the experiments of Section 2 would be that $W_{\chi}$ is purely elastic

$W_{\chi}=W_{\chi}^{\mathrm{e}}$

which would clearly simplify the material formulation (assumption (5) has been applied in (Han \& Nikolov, 2007) for developing the hardness model). Therefore, this case will be also discussed throughout this article.

In this respect, it should be noted that indentation experiments of the elastomer PDMS would indicate that for deformation involving rotation gradients, rate dependence may increase (Tatiraju \& Han, 2010; Alisafaei et al., 2013) with decreasing indentation depth. However, as rate dependence in general is not considered here, the length scale dependent deformation of glassy polymers is assumed to be rate independent in the material formulation for simplicity (see, e.g., Gudmundson, 2006, for a length scale dependent linear viscoelastic model).

As mentioned in Section 2, similar to metals, size effect in polymers is associated with the changes in the higher order displacement gradients and it was experimentally shown that these higher order displacement gradients, and therefore size effects, are significantly of elastic nature in polymers. Also, from the bending experiments of Lam et al. (2003) it should be clear that polymers can also exhibit length scale dependent elastic deformation before reaching any yield limits. If a length scale dependent elastoplastic theory is pursued, such a theory should be also capable to describe length scale dependent deformation before reaching the yield limit. As basis for such a length scale dependent theory, the couple-stress elasticity of Yang et al. (2002) is chosen here as, in addition to its simplicity (it only requires one additional material parameter), it can be also micromechanically motivated for polymers (Nikolov et al., 2007).

\subsection{Basic kinematics}

The continuum kinematics is here defined under the assumption of small deformation. Some glassy 
polymers are known to experience finite strains before reaching the elastic limit and, therefore, a large deformation couple-stress elasto-plasticity theory would be more suitable to predict the behavior of such polymers (see, e.g., Srinivasa \& Reddy, 2013, where a large deformation rotation gradient based model is proposed for elastic materials). There are several possible options of introducing finite strains into elastoplastic formulations (see, e.g., Simo \& Hughes, 1998) into a couple-stress setting. However, the material formulation proposed here is restricted to a small deformation setting for the clarity of the material formulation and to avoid the complexity of the large displacement/strain kinematic setting.

In an infinitesimal deformation setting, the strain tensor $\varepsilon_{i j}$ is defined as the symmetric part of the displacement gradient $u_{i, j}$

$\varepsilon_{i j}=\frac{1}{2}\left(u_{i, j}+u_{j, i}\right)$,

where $\varepsilon_{i j}$ is decomposed into elastic $\varepsilon_{i j}^{\mathrm{e}}$ and plastic $\varepsilon_{i j}^{\mathrm{p}}$ components, i.e.

$\varepsilon_{i j}=\varepsilon_{i j}^{\mathrm{e}}+\varepsilon_{i j}^{\mathrm{p}}$.

Following (Yang et al., 2002), it is assumed that only the symmetric part of the rotation gradient

$\chi_{i j}=\frac{1}{2}\left(\omega_{i, j}+\omega_{j, i}\right)$

enters the constitutive equations, where the symmetric part of the rotation gradient tensor $\chi_{i j}$ can be defined by the infinitesimal rotation vector

$\omega_{i}=\frac{1}{2} e_{i m n} u_{n, m}$

with the displacement vector $u_{i}$ and permutation symbol $e_{i m n}$. With (8), only the rotation gradients are applied here as the higher order displacement gradients. Besides gradients in the rotation, other second order displacement gradients (such as gradients of normal and shear strains) could also be applicable to describe length scale dependent deformation in polymers. However, the rotation gradient model is adopted here as, in addition to its simplicity, this model has successfully captured size effect in elastic deformation of polymers (Lam et al., 2003) and has been also micromechanically motivated in (Nikolov et al., 2007; Han \& Nikolov, 2007).

Assuming a decomposition as in (4), corresponding additive decomposition of the rotation gradient tensor could be motivated

$\chi_{i j}=\chi_{i j}^{\mathrm{e}}+\chi_{i j}^{\mathrm{p}}$.

Assuming $W_{\chi}=W_{\chi}^{\mathrm{e}}$ in Eq. (5) for the glassy polymers, the non-local rotation gradients $\chi_{i j}$ would be of elastic nature and therefore a decomposition of $\chi_{i j}$ into elastic and plastic parts would not be necessary

$\chi_{i j}=\chi_{i j}^{\mathrm{e}}$

where all rotation gradients would be reversible in nature even if local plastic deformation would be present which in turn may actually freeze the non-local stored elastic energy.

\subsection{Power relations}

According to Yang et al. (2002), the internal power per unit volume $P_{\text {int }}$ can be given as 
$P_{\text {int }}=\sigma_{i j} \dot{\varepsilon}_{i j}+m_{i j} \dot{\chi}_{i j}$

where $\sigma_{i j}, m_{i j}, \dot{\varepsilon}_{i j}$ and $\dot{\chi}_{i j}$ are Cauchy stress tensor, couple-stress tensor, strain rate tensor, and rate of the symmetric part of the rotation gradient tensor, respectively.

\subsection{Non-local Clausius-Duhem inequality}

While decomposing or not decomposing $\chi_{i j}$ into elastic and inelastic components (either Eq. (4) or (5)) is irrelevant for the power relation of Section 3.2., for the Clausius-Duhem inequality relation decomposing or not decomposing $\chi_{i j}$ would make quite a difference, so that both cases will be considered in the following.

Additive decomposition of rotation gradients $\chi_{i j}=\chi_{i j}^{\mathrm{e}}+\chi_{i j}^{\mathrm{p}}$. First, the more general case of the additive decomposition of the rotation gradient tensor is considered. The Clausius-Duhem inequality for isothermal processes and homogeneous distribution of the temperature can be stated as

$P_{\text {int }}-\dot{\psi}=\sigma_{i j} \dot{\varepsilon}_{i j}+m_{i j} \dot{\chi}_{i j}-\dot{\psi} \geq 0$,

where $\psi$ denotes the specific Helmholtz free energy function per unit volume. The specific free Helmholz free energy functions is considered to be additively decomposed into elastic and plastic parts

$\psi(t)=\psi^{\mathrm{e}}(t)+\psi^{\mathrm{p}}(t)$

where the plastic part $\psi^{\mathrm{p}}$ is assumed to depend on $\varepsilon_{i j}^{\mathrm{p}}, \chi_{i j}^{\mathrm{p}}$, and other local internal variables and the elastic part $\psi^{\mathrm{e}}$ only on $\varepsilon_{i j}^{\mathrm{e}}$ and $\chi_{i j}^{\mathrm{e}}$. Following (Han \& Nikolov, 2007; Nikolov et al., 2007), the non-local elastic component $W_{\chi}^{\mathrm{e}}$ in (4) is expressed as a function of $\chi_{i j}^{\mathrm{e}}$

$W_{\chi}^{\mathrm{e}}=\frac{\widetilde{K}}{3} \chi_{i j}^{\mathrm{e}} \chi_{i j}^{\mathrm{e}}$

where $\widetilde{K}$ is a material constant that can be micromechanically motivated (Han \& Nikolov, 2007; Nikolov et al., 2007) and is dependent on bending rigidity of the polymer backbone, cross link density, molecular weight, and other molecular properties. In (15) and in the following, it should be clear that summation will only take place over slanted subscripts and not over superscripts 'e' and 'p'.

As mentioned earlier, the non-local elastic component $W_{\chi}^{\mathrm{e}}$ can be physically viewed as a result of interactions between polymer chains and their bending stiffness. When applying classical local elasticity to polymers, the bending stiffness of polymer chains and their interaction are usually assumed to be negligible in a large representative volume of material. However, at small scales, both the bending stiffness of polymer chains and their interactions (with neighboring chains) can become of importance for describing the mechanical behavior of polymers at micro-nano scales. In (Nikolov et al., 2007; Han \& Nikolov, 2007), the non-local elastic component $W_{\chi}^{\mathrm{e}}$ is related to changes in a potential energy (called non-local potential energy hereafter) which can be motivated by similar mechanism as the Frank energy of liquid crystal polymers. The non-local potential energy has two major sources in polymers; (i) rotational entropy of polymer chains, and (ii) interaction between polymer molecules. In amorphous polymers, such a non-local potential energy would not be zero even in the undeformed state from a molecular point of view as the polymer chains are not aligned. By inducing inhomogeneous deformation, this potential is increased. The non-local elastic component $W_{\chi}^{\mathrm{e}}$ is the result of such changes in the non- 
local potential energy and can be mathematically formulated by higher order displacement gradients shown in Eq. (15) or in the other forms. With (15), the elastic deformation work density can be written as

$\psi_{\mathrm{e}}=W_{\varepsilon}^{\mathrm{e}}+W_{\chi}^{\mathrm{e}}=\varepsilon_{i j}^{\mathrm{e}} C_{i j k m}^{\mathrm{e}} \varepsilon_{k m}^{\mathrm{e}}+\frac{\widetilde{K}}{3} \chi_{i j}^{\mathrm{e}} \chi_{i j}^{\mathrm{e}}$,

where $C_{i j k m}^{\mathrm{e}}$ is the elasticity tensor. Following standard work density considerations, the stress tensor $\sigma_{i j}$ and the couple-stress tensor $m_{i j}$ are respectively obtained as

$\sigma_{i j}=\frac{\partial \psi^{\mathrm{e}}}{\partial \varepsilon_{i j}^{\mathrm{e}}}$

and

$m_{i j}=\frac{\partial \psi^{\mathrm{e}}}{\partial \chi_{i j}^{\mathrm{e}}}=\frac{2}{3} \widetilde{K} \chi_{i j}^{\mathrm{e}}$

Thus, Eq. (13) can be rewritten in the following form

$D=\sigma_{i j} \dot{\varepsilon}_{i j}^{\mathrm{p}}+m_{i j} \dot{\chi}_{i j}^{\mathrm{p}}-\dot{\psi}^{\mathrm{p}} \geq 0$

where $D$ denotes the local internal dissipation.

In view of (Han \& Nikolov, 2007), Frank energy type mechanisms (related to rotation gradients in Nikolov et al., 2007) should be also present in elasto-plastic deformation. Following this notion, the elastic nature of $\chi_{i j}$, i.e. $\chi_{i j}^{\mathrm{e}}$, may be less pronounced for thermoplastic polymers with smaller molecular weights as the plastic deformation may relax the related energies by reconfiguration of the chain structure. Generally, such considerations or assumptions are however difficult to examine with molecular simulations as even determining the positions of the polymer atoms in the undeformed configuration presents some formidable challenges. As the deformation in polymers is inherently strongly time dependent, long time ranges have to be applied and because inhomogeneous deformation should be examined, at the current state, meaningful molecular simulations of length scale dependent elasto-plastic deformation in polymers will be arguably very difficult to achieve.

Purely elastic rotation gradients $\chi_{i j}=\chi_{i j}^{\mathrm{e}}$. This case can be of course considered as a special case of the considerations above. In this case (15), (16), (18), (19) can be replaced by

$W_{\chi}=\frac{\widetilde{K}}{3} \chi_{i j} \chi_{i j}$

$\psi_{\mathrm{e}}=W_{\varepsilon}^{\mathrm{e}}+W_{\chi}=\varepsilon_{i j}^{\mathrm{e}} C_{i j k m}^{\mathrm{e}} \varepsilon_{k m}^{\mathrm{e}}+\frac{\widetilde{K}}{3} \chi_{i j} \chi_{i j}$

$m_{i j}=\frac{\partial \psi^{\mathrm{e}}}{\partial \chi_{i j}}=\frac{2}{3} \widetilde{K} \chi_{i j}$

$D=\sigma_{i j} \dot{\varepsilon}_{i j}^{\mathrm{p}}-\dot{\psi}^{\mathrm{p}} \geq 0$

respectively. From Eq. (23) it can be seen that the local plastic deformation is identical to conventional local elasto-plastic material formulations.

It may be worth noting that the glass transition temperature of polymers and correspondingly their elastic moduli are known to increase with the hydrostatic pressure, see (Seltzer et al., 2001) and the 
references therein, which is not reflected in the equations above. As polymers are exposed to higher hydrostatic pressure under nanoindentation (compared to tensile test), the elastic modulus of polymers obtained from nanoindentation is argued to be likely higher than the one obtained from tensile test particularly at high temperatures in (Seltzer et al., 2001). However, the indentation and tensile elastic moduli of polyimide were found to agree well with each other at different temperatures ranging from 20 to $200{ }^{\circ} \mathrm{C}$ ( $\mathrm{Lu}$ et al., 2010).

\subsection{Dissipation and flow rule}

To complete the material formulation, internal variables should be introduced and evolution equations for the plastic terms should be provided which will be described in the following for the both cases where rotation gradient tensor is decomposed and remains fully reversible.

Additive decomposition of rotation gradients $\chi_{i j}=\chi_{i j}^{\mathrm{e}}+\chi_{i j}^{\mathrm{p}}$. Assuming that the plastic part of $\psi$ is dependent on a set of strain-like internal variables $\alpha_{k}, k=1, \ldots, n_{\alpha}$ (where $\alpha_{k}$ can be scalar variables or tensor variables, like back stress related terms, and may also include non-local inelastic terms since $\chi_{i j}^{\mathrm{p}}$ can be viewed as internal variable) with its thermodynamically conjugated stress-like internal variable defined as

$q_{k}=-\frac{\partial \psi^{\mathrm{p}}}{\partial \alpha_{k}}$

the time derivative of the isotropic part of the plastic free energy function $\psi^{\mathrm{p}}$ can be obtained by $\dot{\psi}^{\mathrm{p}}=$ $\sum_{k}\left(-q_{k} \dot{\alpha}_{k}\right)$. Therefore, the local internal dissipation function, $D$, in Eq. (19) can be written as

$D=\sigma_{i j} \dot{\varepsilon}_{i j}^{\mathrm{p}}+m_{i j} \chi_{i j}^{\mathrm{p}}+\sum_{k} q_{k} \dot{\alpha}_{k} \geq 0$

To derive the evolution equations, a convex yield function $f=f\left(\sigma_{i j}, m_{i j}, q_{k}\right)$ should be defined, describing the elastic range of $\sigma_{i j}$ and $m_{i j}$ as $\mathbb{E}=\left\{\left(\sigma_{i j}, m_{i j}, q_{k}\right) \mid f\left(\sigma_{i j}, m_{i j}, q_{k}\right) \leq 0\right\}$. Although metal plasticity theories have been often applied to polymers, the underlying mechanisms of polymers are quite different from metals and can also differ strongly between two different polymers. For instance, many polymers experience yield stress softening after initial yield (see, e.g., Ward \& Sweeney, 2013; Jatin et al., 2014) and the yielding has been also found to be pressure dependent and anisotropic with respect to tension and compression (see, e.g., Argon, 1973; Raghava \& Caddell, 1973; Rottler \& Robbins, 2001; Poulain et al., 2014). Here, plastic deformation is treated in a phenomenological way (for the underlying physics of plastic deformation in polymers, see, e.g., Ward \& Sweeney, 2013). Also, as mentioned above, rate dependence of yielding is not considered here albeit rate dependence of yielding has been shown to be present in polymers (see, e.g., Truss et al., 1984; Liu \& Truss, 1994; Richeton et al., 2005). Even without any higher gradient related terms, there are various different yield function descriptions suggested in the literature. A review or discussion of these yield functions is however not the goal of this article and the interested reader may be referred to, e.g., (Ward \& Sweeney, 2013) and references therein.

As mentioned before, in metals usually length scale dependent deformation is only restricted to plastic deformation and for a wide range of applications, such as indentation, size effects in metals can be successfully modeled by considering $f$ to be dependent on gradients of the plastic strain (see, e.g., Huang et al. 2004; Lee \& Han, 2012). In a general setting, this could be also assumed to be the case for polymers as it has been suggested in (Lam \& Chong, 1999). However, as length scale dependent 
deformation is also present in elastic deformation of polymers, the necessity or significance of the yield function to be dependent on higher order displacement gradient terms (or gradients of strain related terms) is unclear and certainly necessitates additional research and discussion.

For completeness, the evolution equations for $\dot{\varepsilon}_{i j}^{\mathrm{p}}, \dot{\chi}_{i j}^{\mathrm{p}}$, and $\dot{\alpha}_{k}$ should be discussed. Just as in ordinary local plasticity, there would be many possible descriptions. For the sake of simplicity, we assume the postulate of maximum dissipation to be valid and limit ourselves to associative plasticity and evolution equations that obey the normality rule. Correspondingly, we have

$\dot{\varepsilon}_{i j}^{\mathrm{p}}=\dot{\gamma} \frac{\partial f}{\partial \sigma_{i j}}$

$\dot{\chi}_{i j}^{\mathrm{p}}=\dot{\gamma} \frac{\partial f}{\partial m_{i j}}$

$\dot{\alpha}_{k}=\dot{\gamma} \frac{\partial f}{\partial q_{k}}$

for the evolution equations where $\dot{\gamma}$ represents the plastic deformation rate which can be for instance described as in (Simo \& Hughes, 1998)

$\dot{\gamma}=\frac{1}{\eta}\left\langle g\left(f\left(\sigma_{i j}, m_{i j}, q_{k}\right)\right)\right\rangle$,

in which $\langle x\rangle=\frac{1}{2}(x+|x|), g(x)$ is a monotone function with $g(x)=0$ corresponding to $x \leq 0$, and $\eta \in(0, \infty)$ is a viscosity parameter with which the rate-independent limit is obtained for $\eta \rightarrow \infty$. Alternatively, $\dot{\gamma}$ can be considered to be the plastic multiplier in combination of Kuhn Tucker conditions if a truly rate independent formulation is desired (see also below).

- Purely elastic rotation gradients $\chi_{i j}=\chi_{i j}^{\mathrm{e}}$. For this case, the plasticity would be decoupled from the rotation gradients, where the internal dissipation function is described with only local terms

$D=\sigma_{i j} \dot{\varepsilon}_{i j}^{\mathrm{p}}+\sum_{k} q_{k} \dot{\alpha}_{k} \geq 0$

Considering again associative plasticity for simplicity, Eq. (27) is obsolete and the evolution equations are obtained as $\dot{\varepsilon}_{i j}^{\mathrm{p}}=\dot{\gamma} \frac{\partial f}{\partial \sigma_{i j}}$ and $\dot{\alpha}_{k}=\dot{\gamma} \frac{\partial f}{\partial q_{k}}$, where a rate independent formulation could be obtained by introduction of Kuhn-Tucker conditions $\dot{\gamma} \geq 0, f \leq 0, \dot{\gamma} f=0$ and consistency condition $\dot{\gamma} \dot{f}=0$ (if $f=$ 0 ) for $\dot{\gamma}$. It should be noted that these evolution equations are identical to local plasticity models.

Such a material description with purely elastic rotation gradients has been applied by Han \& Nikolov (2007) to propose an indentation depth dependent hardness model (hardness as a function of indentation depth). Recently, based on the Frank energy type mechanism and using the findings of (Nikolov et al., 2007; Han \& Nikolov, 2007) for proposing Eq. (15), a general higher order displacement gradient model has been suggested in (Voyiadjis et al., 2014) where both elastic and plastic parts of rotation and strain gradients contribute to the non-local term $W_{\chi}$. However, to our knowledge, there is no complete elastoplastic material formulation in the literature where the length scale dependent deformation is described elastically which is in contrast to metals where length scale dependent deformation is usually described purely with plastic deformation. Furthermore, our proposed formulation is based on the couple stress 
elasticity of Yang et al., (2002) which contains only one couple stress (in contrast to strain gradient plasticity of Voyiadjis et al., (2014) which contains four different couple stresses) and consequently yields simpler implementations for numerical simulations.

\subsection{Equilibrium equations, boundary conditions, and weak form of equilibrium}

To complete the material formulation, the equilibrium equations and boundary conditions should be stated. As the rotation gradients and the corresponding couple-stresses are not affected by an elastoplastic decomposition of the deformation, the boundary conditions and equilibrium conditions are the same as for the couple-stress elasticity model of Yang et al. (2002). Neglecting couple body forces, the equilibrium equations are given as

$\sigma_{i k, i}-\frac{1}{2} e_{j l k} m_{i j, i l}+b_{k}=0$

where $b_{k}$ is the body force vector per unit volume. Boundary conditions are given with

$n_{j}\left(\sigma_{j k}-\frac{1}{2} e_{j k l} m_{i l, i}\right)-\frac{1}{2} e_{j k l} D_{l}\left(n_{p} n_{q} m_{p q} n_{j}\right)=\bar{t}_{k} \quad$ or $\quad u_{k}=\bar{u}_{k}$

$n_{i} m_{i j}-\left(n_{p} m_{p q} n_{q}\right) n_{j}=\bar{q}_{j} \quad$ or $\quad\left(\delta_{i j}-n_{i} n_{j}\right) \omega_{i}=\bar{\omega}_{j}$

where $D_{j}()=.\left(\delta_{j k}-n_{j} n_{k}\right) \partial(.) / \partial x_{k}$ is the surface gradient operator, $n_{i}$ denotes the unit normal to the outer surface, $t_{k}$ is the surface traction, and $\bar{u}_{k}, \bar{\omega}_{j}, \bar{t}_{k}, \bar{q}_{j}$ are the prescribed values of displacements, rotations, tractions, and moments on their corresponding boundaries, respectively.

The weak form of equilibrium can be stated with body volume $V$ and surface area $S$ as

$\int_{V} \sigma_{i j} \delta \varepsilon_{i j} d V+\int_{V} m_{i j} \delta \chi_{i j} d V-\int_{V} f_{i} \delta u_{i} d V-\int_{S} t_{i} \delta u_{i} d S-\int_{S} q_{i} \delta \omega_{i} d S=0$,

where the surface traction vector $t_{i}$ and couple surface traction vector $q_{i}$ are respectively related to Cauchy stress tensor $\sigma_{i j}$ and couple-stress tensor $m_{i j}$ with $t_{i}=\sigma_{j i} n_{j}$ and $q_{i}=m_{j i} n_{j}$ and the variations in (34) can be given as $\delta \varepsilon_{i j}=\frac{1}{2}\left(\delta u_{i, j}+\delta u_{j, i}\right)$ and $\delta \chi_{i j}=\frac{1}{2}\left(\delta \omega_{i, j}+\delta \omega_{j, i}\right)$ with $\delta \omega_{i, j}=\frac{1}{2} e_{i m n} \delta u_{n, m j}$. The rotation gradient tensor $\chi_{i j}$ in (34) renders the governing differential equation to be of fourth order which requires $C^{1}$ continuity in a displacement based finite element formulation (Garg \& Han, 2013, 2015; Zervos et al., 2001; Shu et al., 1999).

The experiments in Section 2 were discussed to establish a phenomenological basis for the suggested model in Section 3. In metals, there is a great variety of material formulations to model length scale dependent elasto-plastic deformation with second order displacement gradients (see, e.g., McDowell, 2010). Correspondingly, in polymers there would be also various other possible settings than couple stress theories for length scale dependent elasto-plastic deformation like, for instance, micropolar theories (see, e.g., Hard af Segerstad et al., 2009 or Mora and Waas, 2007). The above suggested constitutive framework is based on the rather simple rotation gradient elasticity model (Yang et al., 2002) that can be micromechanically motivated for polymers (Nikolov et al., 2007) and requires only one additional variable for isotropic materials yielding a rather simple framework for elasto-plastic polymeric materials. 


\section{Elasto-plastic simulation of wedge indentation}

To illustrate the effect of rotation gradients on the mechanical behavior of polymers at micro- to nanometer length scales, a wedge indentation of epoxy is studied here. To this aim, a finite element framework is presented for the suggested elasto-plastic couple stress theory followed by the simulation results.

Finite element framework. The appearance of the rotation gradient tensor $\chi_{i j}$ in the total deformation energy density (34) requires $C^{1}$ continuity of the displacements in the corresponding finite element approach (Fischer et al., 2011; Papanicolopulos et al., 2009; Petra \& Pittman, 1994; Zervos et al., 2001). Due to the difficulty of imposing $C^{1}$ continuity in problems containing second derivatives of field variables, several alternative approaches have been suggested in the literature (Shu et al., 1999; Amanatidou \& Arvas, 2002; Xia \& Hutchinson, 1996; Shu \& Fleck, 1998; Adachi et al., 1998; Askes and Gutierrez, 2006; Askes et al., 2000; Askes \& Aifantis, 2002; Tang et al., 2003; Wells et al., 2004).

Here, to relax the $C^{1}$ continuity requirement and avoid large number of DOF, similar to (Garg \& Han, 2013), the rotation vector $\phi_{i}$ is treated as extra nodal variables (in addition to displacements $u_{i}$ ) and is constrained to the rotation vector $\omega_{i}$ (determined from the displacements $u_{i}$ ) by a penalty approach, i.e., the rotation vector $\omega_{i}$ is replaced by $\phi_{i}$. Therefore, Eq. (34) is rewritten in the following form

$\int_{V} \sigma_{i j} \delta \varepsilon_{i j} d V+\int_{V} m_{i j} \delta \chi_{i j}^{\phi} d V+P \int_{V} \delta \alpha_{i} \alpha_{i} d V-\int_{V} f_{i} \delta u_{i} d V-\int_{S} t_{i} \delta u_{i} d S-\int_{S} q_{i} \delta \phi_{i} d S=0$

where $\alpha_{i}=\phi_{i}-\omega_{i}, \delta \alpha_{i}=\left(\delta \phi_{i}-\frac{1}{2} e_{i j k} \delta u_{k, j}\right), \delta \chi_{i j}^{\phi}=\frac{1}{2}\left(\delta \phi_{i, j}+\delta \phi_{j, i}\right), m_{i j}=\frac{2}{3} \widetilde{K} \chi_{i j}^{\phi}$ and $P$ is the penalty parameter. More details of finite element approach are given in Appendix (A) for elasto-plastic couple stress theory discussed below.

Algorithmic treatment of elasto-plastic constitutive equations. As yielding of polymers is known to be pressure dependent, the yield function $f$ could be a function of pressure. Various pressure dependent yield function descriptions have been suggested in the literature (see, e.g., Ward \& Sweeney, 2013). Here, the Drucker-Prager yield criterion is applied to include pressure dependence of yielding in epoxy (see, e.g., Seltzer et al., (2011); Bardia \& Narasimhan, (2006); Du Bois et al., (2006) for the use of Drucker-Prager model in polymers). As mentioned earlier, the yield function of polymers, similar to metals, have been considered in (Lam \& Chong, 1999; Voyiadjis et al., 2014; Swaddiwudhipong et al., 2005 ) to be dependent on the higher order displacement gradients at micro-nano scales. Although it may be assumed to be the case for polymers in a general setting, here it is assumed for simplicity that yielding function of epoxy is independent of higher order displacement gradients and only the pressure dependence of yield function is taken into account by using Drucker-Prager yield criterion. In DruckerPrager criterion, the influence of a hydrostatic stress component on yielding is introduced by an additional term in the von Mises yield criterion, $\sqrt{J_{2}^{\prime}}=k$, as follows

$\alpha J_{1}+\sqrt{J_{2}^{\prime}}=k^{\prime} \quad$,

where $J_{2}^{\prime}=\frac{1}{2} \sigma_{i j}^{\prime} \sigma_{i j}^{\prime}$ is the second deviatoric stress invariant, $\sigma_{i j}^{\prime}=\sigma_{i j}-\frac{1}{3} \delta_{i j} \sigma_{k k}$ is the deviatoric stress, $\delta_{i j}$ is the Kronecker delta, $k=\frac{1}{\sqrt{3}} \sigma_{\mathrm{Y}}$ is an empirical material parameter, $\sigma_{\mathrm{Y}}$ is the yield stress, and $J_{1}=$ $\sigma_{i i}$ is the first stress invariant. Also, the material constants $\alpha$ and $k^{\prime}$ are given as follows when the Drucker-Prager circular cone yield surface circumscribes the Mohr-Coulomb hexagonal pyramid 
$\alpha=\frac{2 \sin \phi}{\sqrt{3}(3-\sin \phi)} \quad, \quad k^{\prime}=\frac{6 c \cos \phi}{\sqrt{3}(3-\sin \phi)}$

where the internal friction angle $\phi$ and the cohesion intercept $c$ are assumed to be $20^{\circ}$ and $140 \mathrm{MPa}$ in this example, respectively. Linear isotropic hardening, i.e.,

$\frac{d \bar{\sigma}}{d \bar{\varepsilon}_{\mathrm{p}}}=H$

is considered here where $\bar{\sigma}$ is the effective stress $\left(\bar{\sigma}=\alpha J_{1}+\sqrt{J_{2}^{\prime}}\right.$ for Drucker-Prager yield criterion and $\bar{\sigma}=\sqrt{3 J_{2}^{\prime}}$ for Von Mises yield criterion), $\bar{\varepsilon}_{\mathrm{p}}$ is the effective plastic strain, and $H$ is the hardening parameter (the slope of flow curve, i.e., local slope of the uniaxial stress-plastic strain curve) which is assumed to be $100 \mathrm{MPa}$. The strain increment $d \varepsilon_{i j}$ is decomposed into elastic and plastic components as $d \varepsilon_{i j}=d \varepsilon_{i j}^{\mathrm{e}}+d \varepsilon_{i j}^{\mathrm{p}}$. The elastic strain increment $d \varepsilon_{i j}^{\mathrm{e}}$ (related to stress increment by $d \sigma_{i j}=C_{i j k l} d \varepsilon_{k l}^{\mathrm{e}}$ ) can be also formulated in the following form to be related to the deviatoric and hydrostatic components of stress increment $d \sigma_{i j}$ (respectively $d \sigma_{i j}^{\prime}$ and $d \sigma_{k k}$ )

$d \varepsilon_{i j}^{\mathrm{e}}=\frac{d \sigma_{i j}^{\prime}}{2 \mu}+\frac{(1-2 v)}{E} \delta_{i j} d \sigma_{k k} \quad$,

where $C_{i j k l}=\lambda \delta_{i j} \delta_{k l}+\mu\left(\delta_{i k} \delta_{j l}+\delta_{i l} \delta_{j k}\right)$ is the elasticity tensor with $\lambda$ and $\mu$ being Lame constants. The plastic strain increment $d \varepsilon_{i j}^{\mathrm{p}}$ is related to the yield function $f$ by the associated theory of plasticity

$d \varepsilon_{i j}^{\mathrm{p}}=d \lambda \frac{\partial f}{\partial \sigma_{i j}} \quad$,

where $d \lambda$ is the plastic multiplier which can be obtained as follows after onset of plastic yielding $(d \lambda=0$ before the onset of plastic yielding)

$d \lambda=\frac{\boldsymbol{n}^{\mathrm{T}} \mathbf{C} d \boldsymbol{\varepsilon}}{\boldsymbol{n}^{\mathrm{T}} \mathbf{C} \boldsymbol{n}+H}$

in which $\boldsymbol{n}=\partial f / \partial \boldsymbol{\sigma}$ is the flow vector $\left(\boldsymbol{n}=a_{\mathrm{v}}\left\{\sigma_{x}^{\prime}, \sigma_{y}^{\prime}, \sigma_{z}^{\prime}, 2 \tau_{y z}, 2 \tau_{z x}, 2 \tau_{x y}\right\}^{\mathrm{T}}\right.$ with $a_{\mathrm{v}}=0.5 \sqrt{3 / J_{2}^{\prime}}$ for von Mises yield criterion, and $\boldsymbol{n}=a_{\mathrm{d}}\left\{\sigma_{x}^{\prime}+2 \alpha \sqrt{J_{2}^{\prime}}, \sigma_{y}^{\prime}+2 \alpha \sqrt{J_{2}^{\prime}}, \sigma_{z}^{\prime}+2 \alpha \sqrt{J_{2}^{\prime}}, 2 \tau_{y z}, 2 \tau_{z x}, 2 \tau_{x y}\right\}^{\mathrm{T}}$ with $a_{\mathrm{d}}=0.5 \sqrt{1 / J_{2}^{\prime}}$ for Drucker-Prager yield criterion), $\sigma=\left\{\sigma_{x}, \sigma_{y}, \sigma_{z}, \tau_{y z}, \tau_{z x}, \tau_{x y}\right\}^{\mathrm{T}}$ is the stress vector, $\boldsymbol{\varepsilon}=\left\{\varepsilon_{x}, \varepsilon_{y}, \varepsilon_{z}, 2 \varepsilon_{y z}, 2 \varepsilon_{z x}, 2 \varepsilon_{x y}\right\}^{\mathrm{T}}$ is the strain vector, and $\mathbf{C}$ is the elasticity matrix. The stress increment $d \boldsymbol{\sigma}$ is related to the strain increment $d \boldsymbol{\varepsilon}$ by $d \boldsymbol{\sigma}=\mathbf{C}_{\mathrm{ep}} d \boldsymbol{\varepsilon}$ where the elasto-plastic continuum tangent matrix $\mathbf{C}_{\mathrm{ep}}$ can be determined as follows (Dunne \& Petrinic, 2005)

$\mathbf{C}_{\mathrm{ep}}=\mathbf{C}-\frac{\mathbf{C} \boldsymbol{n} \otimes \mathbf{C} \boldsymbol{n}}{\boldsymbol{n}^{\mathrm{T}} \mathbf{C} \boldsymbol{n}+H}$

where $\otimes$ represents the dyadic product of two vectors. The reader is referred to (de Souza Neto et al., 2008; Szabo \& Kossa, 2012) for determination of the consistent tangent matrix associated with the Drucker-Prager yield criterion with isotropic hardening, and also to (Li \& Tang, 2005) for consistent tangent matrix in a Drucker-Prager elasto-plastic Cosserat continuum model. The continuum tangent matrix (42) can be used in conjunction with the return-mapping scheme $\boldsymbol{\sigma}^{(\mathrm{r})}=\boldsymbol{\sigma}^{(\mathrm{r}-1)}+\mathbf{C} d \boldsymbol{\varepsilon}^{(\mathrm{r})}-d \lambda \mathbf{C} \boldsymbol{n}$ 
where the term ( $d \lambda \mathbf{C} \boldsymbol{n})$ is the plastic corrector (return vector) to satisfy the yield criterion and $\mathbf{C} d \boldsymbol{\varepsilon}^{(\mathrm{r})}$ is the trial elastic stress increment $d \sigma_{\mathrm{e}}{ }^{(\mathrm{r})}$ at $\mathrm{r}^{\text {th }}$ iteration. As an alternative, the consistent tangent matrix can be also used together with an implicit elastic predictor/return-mapping algorithm for the DruckerPrager yield criterion (see, e.g., de Souza Neto et al., 2008 for details).

Numerical results. The wedge indenter tip is modeled as a rigid body with a half angle $\alpha=70.3^{\circ}$ and a tip roundness $r=50 \mathrm{~nm}$. Due to the symmetry condition, only half of the specimen and indenter are modeled. All degrees of freedom are constrained to be zero at the bottom of the sample. Also, all nodes on the left side of the sample are constrained as $u_{3}=0$ (zero displacement in $x_{3}$ direction) and $\phi_{3}=0$ (zero rotation about $x_{3}$ axis). Eight node quadrilateral elements with selective reduced integration (using four gauss points) are used as shown in Fig. 12 (a). In all simulations, 8400 (140 by 60) elements with finer meshes beneath the indenter tip are applied as depicted in Fig, 12 (b). Simulation with denser meshes resulted in negligible changes in the simulation results. The elastic modulus of the epoxy sample, $E$, is assumed to be $3.0 \mathrm{GPa}$ which is in the range of the elastic modulus of epoxy determined by the nanoindentation experiments $\left(E_{\mathrm{B}}^{\mathrm{O} \& \mathrm{P}}\right.$ at large depths, $E_{\mathrm{sphere}}^{\mathrm{Hertz}}$ and $\left.E_{\mathrm{sphere}}^{\mathrm{O} \& \mathrm{P}}\right)$. Poisson's ratio of the epoxy sample, $v$, is assumed to be 0.35 as in the evaluation of nanoindentation experiments. The same $\widetilde{K}=$ $0.116 \mathrm{~N}$ and penalty parameter $P=10^{12}$ are used in the indentation simulations as in the micro beam bending simulations of Fig. 1 (Garg \& Han, 2013).

Fig. 13 illustrates the results of wedge indentation simulations where the normalized force is plotted with respect to $h_{\max }$. In Fig. 13, the normalized force is the ratio of the reaction force with including the rotation gradient effects to the reaction force without the presence of rotation gradients. As the hardness is proportional to the applied force $F$, the normalized force plotted in Fig. 13 illustrates the changes in the stiffness with $h_{\max }$. It can be seen in Fig. 13 that the normalized force significantly increases with

decreasing $h_{\text {max }}$ from $40 \mu \mathrm{m}$ to $100 \mathrm{~nm}$ indicating the stiffening of epoxy with decreasing indentation depth as observed in the experiments. In Fig. 14, the effective stress $\alpha J_{1}+\sqrt{J_{2}^{\prime}}$ is plotted for $h_{\max }=100$ $\mathrm{nm}$ with and without considering rotation gradients. In addition to the stiffening effect, it is seen in Fig. 14 that including rotation gradients in the simulations will also affect the distribution of effective stress. The non-zero components of rotation gradients $\left(\chi_{13}\right.$ and $\left.\chi_{23}\right)$ for the same indentation depth $h_{\max }=100$ $\mathrm{nm}$ are demonstrated in Fig. 15, respectively.

\section{Discussions}

With the experiments of Section 2, it should be clear that the length scale dependent deformation in polymers is, at least in parts, strongly elastic. On this conclusion, an elasto-plastic material formulation setting at small strains has been suggested that is relatively simple. Rate effects which are often quite pronounced in polymers have been neglected in the formulation for simplicity and in many aspects the suggested constitutive framework is rather unspecific as for instance a specific yield function has not been provided. It should be however noted that while abundant experimental data on length scale dependent deformation is available for metals and higher gradient concepts in metals can be compared with discrete dislocation dynamics simulations (e.g. Bittencourt et al., 2003) or even molecular simulations, for polymers experiments studying length scale dependent deformation at the micron to submicron scale are rather scarce and the few molecular studies are less compelling. Correspondingly, some of the assumptions that have been made for the suggested material formulation framework may have to be 
restricted to a smaller subset of polymers or generally abandoned. Also, we have here opted for simplicity rather than generality as we intended to use this material formulation setting for numerical simulations of the indentation experiments discussed in Section 2. To describe the plastic deformation of polymers, the three evolution equations (26)-(28) have been adopted from the (non-local) classical plasticity model. As an alternative, local models (micromechanically motivated for the local elastoplastic deformation of polymers) could be also used for the evolution equations. However, these local models may be only applied for a specific class of polymers.

Utilizing a Cosserat continuum framework and assuming small deformations, a much more elaborated strain gradient formulation has been recently developed for amorphous polymers (Voyiadjis et al., 2014) where both elastic and plastic parts of the higher order displacement gradients contribute to the non-local term $W_{\chi}$ presented in Eq. (3). The elastic length scale dependence in (Voyiadjis et al., 2014) is described similarly as in (Nikolov et al., 2007) and also in the present article although the material formulation in (Voyiadjis et al., 2014) contains four different couple stresses. A different notion has been pursued in the strain gradient plasticity models of (Lam \& Chong, 1999; Swaddiwudhipong et al., 2005) where the length scale dependent deformation of polymers is only attributed to strain gradients of plastic deformations. It should be also mentioned that, similar to metals, the yield function in polymers is usually assumed to be dependent on the higher order gradients of displacements (Lam \& Chong, 1999; Voyiadjis et al., 2014; Swaddiwudhipong et al., 2005). Although it may be assumed to be the case for polymers in a general setting, to the authors' knowledge, there is no experimental study available in the literature indicating the dependence of the yield function in polymers on higher order displacement gradients. In this respect, it may be emphasized that while the indentation depth dependent hardness can be modeled with length scale dependence restricted to plastic deformation, the indentation depth dependent elastic unloading observed in the experiments (as discussed in Section 2) cannot be described with such a formulation.

Experimental data provided by elasto-plastic indentations of thermosetting and thermoplastic polymers indicate that, unlike in metals, the higher order displacement gradients, as the origin of length scale dependent deformation in polymers, are to a significant extend elastic. Therefore, in modeling of length scale dependent deformation in polymers, the elastic length scale dependence observed in experiments before the onset of plastic deformation (Lam et al., 2003) should not be ignored. Similar to (Voyiadjis et al., 2014), only small deformation kinematics are considered in this study for simplicity. As many polymers experience large strains before reaching the elastic limit, a length scale dependent finite strain elasto-plastic formulation would be desirable. To this end, non-local formulations proposed for elastic materials with large deformations (Srinivasa \& Reddy, 2013; Hard af Segerstad et al., 2009) may serve as a basis to extend the present elasto-plastic framework to large deformation kinematics.

The indentation tests presented in Section 2 with two tip geometries (Berkovich and spherical tips) illustrate the importance of higher order displacement gradients on the length scale dependent deformation of polymers. However, in addition to the higher order displacement gradient based models (Lam \& Chong, 1999; 2000; 2001; Chong \& Lam, 1999; Han \& Nikolov, 2007; Nikolov et al., 2007; Voyiadjis et al., 2014; Swaddiwudhipong et al., 2005), there are also other rationales suggested in the literature as origin of size effect in polymers including surface effects (Zhang \& Xu, 2002; Zhang et al., 2004), friction (Lim \& Chaudhri, 2006), change in the material properties through thickness (Briscoe et al., 1998) and others (Charitidis, 2011).

It should be mentioned that although surface roughness, surface effect/stress, friction, change in the glass transition temperature, adhesion, tip bluntness/imperfection, and confined molecular motion 
interfacial region (formation of an interfacial region of confined molecular motion between the surface and probe by contact loading) can cause indentation size effects at shallow indentation depths, they can neither explain size effects observed at larger indentation depths with sharp indenters (Alisafaei et al., 2014; Dutta et al., 2004) nor length scale dependent deformation in micro-beam bending tests (Lam et al., 2003; McFarland \& Colton, 2005). Also, material inhomogeneity and/or change in the material properties cannot be the only source of size effect in polymers as the measured indentation elastic modulus of epoxy (obtained by a spherical tip) remains constant with indentation depth while the indentation hardness (determined by a sharp Berkovich tip) increases significantly with decreasing depth (Alisafaei et al., 2014). The influence of material inhomogeneity (change in the material properties through thickness) and surface roughness on the length scale dependent deformation of polymers have been observed in (Balta Calleja et al., 2004) where a significant decrease in the hardness of PS has been reported by removing the top surface. However, the hardness still increased with decreasing indentation depth, even after removing the top layers of the surface, indicating that material inhomogeneity and surface roughness cannot be the only sources of size effect in polymers. In addition, investigating the effect of material inhomogeneity on the length scale dependent deformation of epoxy (Lam et al., 2003) showed that the significant increase in the bending stiffness of epoxy micro-beams is not associated with the presence of stiff surface layers.

\section{Conclusion}

Length scale dependent deformation has been experimentally observed in both metals and polymers. Similar to metals, indentation tests with different tip geometries shown that the length scale dependent deformation in polymers is also associated with an increase in higher order displacement gradients with decreasing length scale. However, experimental data discussed in this study reveals that the nature of such higher order displacement gradients, and subsequently the nature of length scale dependent deformation, in polymers are quite different from metals. In contrast to metals where length scale dependent deformation is associated with non-uniform plastic deformation, in polymers length scale dependent elasto-plastic deformation is, at least in parts, of elastic nature. Corresponding to these findings, a rather simple length scale dependent elasto-plastic couple stress material formulation framework was presented where the rotation gradient tensor has a reversible component. While this framework was formulated in a small strain kinematics setting for simplicity, a formulation in finite strain kinematics could be also derived applying the suggested concepts by using finite strain kinematics elasticity formulations of the literature as a basis.

\section{Acknowledgments}

The support of this work through the National Science Foundation, Grants CMMI 1102764 and CMMI 1126860 , is highly appreciated.

\section{Appendix A}

Based on the developed couple stress theory, a two dimensional plane strain finite element formulation (Garg \& Han, 2013) is here applied to provide illustrative numerical simulations. For plane strain 
problems, the displacement in $x_{3}$ direction, $u_{3}$, the rotation components about $x_{1}$ and $x_{2}$ axes, $\phi_{1}$ and $\phi_{2}$, and correspondingly $\varepsilon_{i 3}$ (for $i=1,2,3$ ) are zero. Thus, each node has three degrees of freedom including $u_{1}$ (displacement in $x_{1}$ direction), $u_{2}$ (displacement in $x_{2}$ direction) and $\phi_{3}$ (rotation about $x_{3}$ axis). The vector $\boldsymbol{\eta}=\left\{\begin{array}{lll}u_{1} & u_{2} & \phi_{3}\end{array}\right\}^{\mathrm{T}}$ containing these three degrees of freedom can be interpolated as $\boldsymbol{\eta}=\mathbf{N}_{\mathrm{e}} \boldsymbol{\eta}_{\mathrm{e}}$ where $\mathbf{N}_{\mathrm{e}}$ is a 3-by-3n matrix ( $n$ is the number of nodes per element) containing the Lagrangian shape functions and $\boldsymbol{\eta}_{\mathrm{e}}$ is a vector containing the element nodal degrees of freedom obtained by

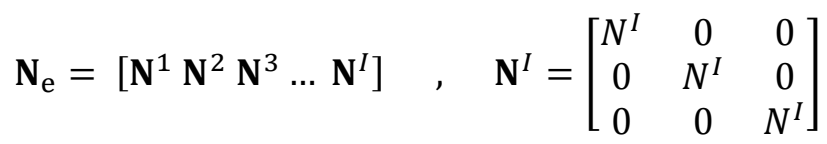

and

$\boldsymbol{\eta}_{\mathrm{e}}=\left\{\boldsymbol{\eta}_{\mathrm{e}}^{1} \boldsymbol{\eta}_{\mathrm{e}}^{2} \boldsymbol{\eta}_{\mathrm{e}}^{3} \ldots \boldsymbol{\eta}_{\mathrm{e}}^{I}\right\}^{\mathrm{T}}, \quad \boldsymbol{\eta}_{\mathrm{e}}^{I}=\left\{\begin{array}{lll}u_{1}^{I} & u_{2}^{I} & \phi_{3}^{I}\end{array}\right\}$

in which $I$ is the number of the element node $(I=1,2,3, \ldots, n)$. The non-zero strain components can be obtained in the vector form as

$\left\{\begin{array}{c}\varepsilon_{11} \\ \varepsilon_{22} \\ 2 \varepsilon_{12}\end{array}\right\}=\left[\begin{array}{ccc}\frac{\partial}{\partial x_{1}} & 0 & 0 \\ 0 & \frac{\partial}{\partial x_{2}} & 0 \\ \frac{\partial}{\partial x_{2}} & \frac{\partial}{\partial x_{1}} & 0\end{array}\right]\left\{\begin{array}{l}u_{1} \\ u_{2} \\ \phi_{3}\end{array}\right\} \quad$ with $\quad \mathbf{D}_{\varepsilon}=\left[\begin{array}{ccc}\frac{\partial}{\partial x_{1}} & 0 & 0 \\ 0 & \frac{\partial}{\partial x_{2}} & 0 \\ \frac{\partial}{\partial x_{2}} & \frac{\partial}{\partial x_{1}} & 0\end{array}\right]$

or equivalently $\boldsymbol{\varepsilon}=\mathbf{D}_{\varepsilon} \boldsymbol{\eta}=\mathbf{D}_{\varepsilon} \mathbf{N}_{\mathrm{e}} \boldsymbol{\eta}_{\mathrm{e}}=\mathbf{B} \boldsymbol{\eta}_{\mathrm{e}}$ where $\mathbf{B}=\mathbf{D}_{\varepsilon} \mathbf{N}_{\mathrm{e}}$ is a strain-displacement operator matrix. Note that in the vector form (Voigt notation) the shear stress components are engineering shears which are twice the tensorial shear stress components. Similarly, the non-zero components of the symmetric rotation gradient tensor $\chi_{i j}^{\phi}$ can be obtained by

$\left\{\begin{array}{c}\chi_{13}^{\phi} \\ \chi_{23}^{\phi}\end{array}\right\}=\left[\begin{array}{lll}0 & 0 & \frac{1}{2} \frac{\partial}{\partial x_{1}} \\ 0 & 0 & \frac{1}{2} \frac{\partial}{\partial x_{2}}\end{array}\right]\left\{\begin{array}{l}u_{1} \\ u_{2} \\ \phi_{3}\end{array}\right\} \quad$ with $\quad \mathbf{D}_{\chi}=\left[\begin{array}{lll}0 & 0 & \frac{1}{2} \frac{\partial}{\partial x_{1}} \\ 0 & 0 & \frac{1}{2} \frac{\partial}{\partial x_{2}}\end{array}\right]$

or equivalently $\chi_{\mathrm{e}}^{\phi}=\mathbf{D}_{\chi} \boldsymbol{\eta}=\mathbf{D}_{\chi} \mathbf{N}_{\mathrm{e}} \boldsymbol{\eta}_{\mathrm{e}}=\mathbf{Q} \boldsymbol{\eta}_{\mathrm{e}}$ in which $\mathbf{Q}=\mathbf{D}_{\chi} \mathbf{N}_{\mathrm{e}}$ is a rotation gradient-displacement operator matrix. Also, considering that in plane strain problems $\phi_{1}=\phi_{2}=0$, the only non-zero component of $\alpha_{i}$ is denoted as $\alpha=\alpha_{3}$ which is determined by

$\alpha=\left\{\begin{array}{lll}\frac{1}{2} \frac{\partial}{\partial x_{2}} & -\frac{1}{2} \frac{\partial}{\partial x_{1}} & 1\end{array}\right\}\left\{\begin{array}{l}u_{1} \\ u_{2} \\ \phi_{3}\end{array}\right\} \quad$ with $\quad \mathbf{D}_{\alpha}=\left\{\begin{array}{lll}\frac{1}{2} \frac{\partial}{\partial x_{2}} & -\frac{1}{2} \frac{\partial}{\partial x_{1}} & 1\end{array}\right\}$

or equivalently $\alpha=\mathbf{D}_{\alpha} \boldsymbol{\eta}=\mathbf{D}_{\alpha} \mathbf{N}_{\mathrm{e}} \boldsymbol{\eta}_{\mathrm{e}}=\mathbf{S} \boldsymbol{\eta}_{\mathrm{e}}$ where $\mathbf{S}=\mathbf{D}_{\alpha} \mathbf{N}_{\mathrm{e}}$ is a constraint-related operator matrix. Also, the displacement vector $\boldsymbol{u}=\left\{\begin{array}{ll}u_{1} & u_{2}\end{array}\right\}^{\mathrm{T}}$ and rotation component $\phi_{3}$ can be interpolated as

$\boldsymbol{u}=\mathbf{N}_{\mathrm{e}, \mathrm{u}} \boldsymbol{\eta}_{\mathrm{e}} \quad$ and $\quad \phi_{3}=\mathbf{N}_{\mathrm{e}, \phi} \boldsymbol{\eta}_{\mathrm{e}}$

where $\quad \mathbf{N}_{\mathrm{e}, \mathrm{u}}=\left[\mathbf{N}_{\mathrm{u}}^{1} \mathbf{N}_{\mathrm{u}}^{2} \mathbf{N}_{\mathrm{u}}^{3} \ldots \mathbf{N}_{\mathrm{u}}^{I}\right] \quad, \quad \mathbf{N}_{\mathrm{u}}^{I}=\left[\begin{array}{ccc}N^{I} & 0 & 0 \\ 0 & N^{I} & 0\end{array}\right]$

$$
\mathbf{N}_{\mathrm{e}, \phi}=\left[\mathbf{N}_{\phi}^{1} \mathbf{N}_{\phi}^{2} \mathbf{N}_{\phi}^{3} \ldots \mathbf{N}_{\phi}^{I}\right] \quad, \quad \mathbf{N}_{\phi}^{I}=\left[\begin{array}{lll}
0 & 0 & N^{I}
\end{array}\right]
$$

Substituting $=\mathbf{B} \delta \boldsymbol{\eta}_{\mathrm{e}}, \delta \chi_{\mathrm{e}}^{\phi}=\mathbf{Q} \delta \boldsymbol{\eta}_{\mathrm{e}}, \delta \alpha=\mathbf{S} \delta \boldsymbol{\eta}_{\mathrm{e}}, \delta \boldsymbol{u}=\mathbf{N}_{\mathrm{e}, \mathrm{u}} \delta \boldsymbol{\eta}_{\mathrm{e}}, \delta \phi_{3}=\mathbf{N}_{\mathrm{e}, \phi} \delta \boldsymbol{\eta}_{\mathrm{e}}$ into Eq. (35) and 
taking $\delta \boldsymbol{\eta}_{\mathrm{e}}^{\mathrm{T}}$ out of the integral, we have

$\int_{V}\left[\mathbf{B}^{\mathrm{T}} \boldsymbol{\sigma}+\frac{8 \widetilde{K}}{3}\left(\mathbf{Q}^{\mathrm{T}} \mathbf{Q}\right) \boldsymbol{\eta}_{\mathrm{e}}+P\left(\mathbf{S}^{\mathrm{T}} \mathbf{S}\right) \boldsymbol{\eta}_{\mathrm{e}}\right] d V-\prod_{\text {ext }}=0$

in which $\prod_{\text {ext }}=\int_{V} \mathbf{N}_{\mathrm{e}, \mathrm{u}}^{\mathrm{T}} \boldsymbol{f} d V+\int_{S} \mathbf{N}_{\mathrm{e}, \mathrm{u}}^{\mathrm{T}} \boldsymbol{t} d S+\int_{S} \mathbf{N}_{\mathrm{e}, \phi}^{\mathrm{T}} \boldsymbol{q} d S$. Since $\chi_{i j}^{\phi}$ is a symmetric matrix, $\chi_{13}^{\phi}=$

$\chi_{31}^{\phi}$ and $\chi_{23}^{\phi}=\chi_{32}^{\phi}$, the coefficient of the second term in Eq. (35), $2 \widetilde{K} / 3$, should be multiplied by four shown in Eq. (A9). For nonlinear problems, Eq. (A9) should be generally solved iteratively. Therefore, the residual vector $\boldsymbol{\psi}$ is introduced as

$\boldsymbol{\psi}=\int_{V}\left[\mathbf{B}^{\mathrm{T}} \boldsymbol{\sigma}+\frac{8 \widetilde{K}}{3}\left(\mathbf{Q}^{\mathrm{T}} \mathbf{Q}\right) \boldsymbol{\eta}_{\mathrm{e}}+P\left(\mathbf{S}^{\mathrm{T}} \mathbf{S}\right) \boldsymbol{\eta}_{\mathrm{e}}\right] d V-\prod_{\text {ext }} \neq 0$

for which its tangent (element tangential stiffness matrix) can be determined in the incremental form as

$d \boldsymbol{\psi}=\int_{V}\left[\mathbf{B}^{\mathrm{T}} d \boldsymbol{\sigma}+\frac{8 \widetilde{K}}{3}\left(\mathbf{Q}^{\mathrm{T}} \mathbf{Q}\right) d \boldsymbol{\eta}_{\mathrm{e}}+P\left(\mathbf{S}^{\mathrm{T}} \mathbf{S}\right) d \boldsymbol{\eta}_{\mathrm{e}}\right] d V-\prod_{\mathrm{ext}}^{\prime} \quad$,

where $\prod_{\mathrm{ext}}^{\prime}=\int_{V} \mathbf{N}_{\mathrm{e}, \mathrm{u}}^{\mathrm{T}} d \boldsymbol{f} d V+\int_{S} \mathbf{N}_{\mathrm{e}, \mathrm{u}}^{\mathrm{T}} d \boldsymbol{t} d S+\int_{S} \mathbf{N}_{\mathrm{e}, \phi}^{\mathrm{T}} d \boldsymbol{q} d S$. In elasto-plastic problems, the stiffness of material is continuously varying where the increment in stress $d \boldsymbol{\sigma}$ is related to the increment in strain $d \boldsymbol{\varepsilon}$ by the elasto-plastic tangent matrix $\mathbf{C}_{\mathrm{ep}}$ as $d \boldsymbol{\sigma}=\mathbf{C}_{\mathrm{ep}} d \boldsymbol{\varepsilon}$

$d \boldsymbol{\psi}=\int_{V}\left[\mathbf{B}^{\mathrm{T}} \mathbf{C}_{\mathrm{ep}} \mathbf{B}+\frac{8 \widetilde{K}}{3}\left(\mathbf{Q}^{\mathrm{T}} \mathbf{Q}\right)+P\left(\mathbf{S}^{\mathrm{T}} \mathbf{S}\right)\right] d \boldsymbol{\eta}_{\mathrm{e}} d V-\prod_{\mathrm{ext}}^{\prime}$

From (A12), the element tangential stiffness matrix $\mathbf{K}_{\mathrm{e}}$ can be calculated in natural coordinates $\xi_{i} \in[-$ $1,+1]$ by Gauss-Lagendre numerical integration as

$\mathbf{K}_{\mathrm{e}}=\int_{-1}^{1} \int_{-1}^{1}\left[\left(\mathbf{B}^{\mathrm{T}} \mathbf{C}_{\mathrm{ep}} \mathbf{B}\right)+\frac{8 \widetilde{K}}{3}\left(\mathbf{Q}^{\mathrm{T}} \mathbf{Q}\right)+P\left(\mathbf{S}^{\mathrm{T}} \mathbf{S}\right)\right]|\mathbf{J}| h d \xi_{1} d \xi_{2}$

where $h$ is the thickness equal to 1 for plane strain problems and $|\mathbf{J}|$ the determinant of Jacobi matrix

$\mathbf{J}=\left[\begin{array}{ll}\frac{\partial x_{1}}{\partial \xi_{1}} & \frac{\partial x_{2}}{\partial \xi_{1}} \\ \frac{\partial x_{1}}{\partial \xi_{2}} & \frac{\partial x_{2}}{\partial \xi_{2}}\end{array}\right]$.

As the rotation gradients do not enter the evolution equations and the Kuhn-Tucker conditions, the stress update algorithm of associated flow rules is identical to those described in classical plasticity theories, see Simo \& Hughes (1998), to which the interested reader is referred to.

\section{References}

Adachi, T., Tomita, Y., Tanaka, M., 1998. Computational simulation of deformation behavior of 2D-lattice continuum. Int. J. Mech. Sci. 40, 857-866.

Alisafaei, F., Han, C.-S., 2015. Indentation depth dependent mechanical behavior in polymers. Adv. Cond. Mat. Phys., 2015, 391579 (20 pages). 
Alisafaei, F., Han, C.-S., Lakhera, N., 2014. Characterization of indentation size effects in epoxy. Polym. Test. 40, 70-78.

Alisafaei, F., Han, C.-S., Sanei, S.H.R., 2013. On the time and depth dependence of hardness, dissipation and stiffness in polydimethylsiloxane. Polym. Test. 32, 1220-1228.

Amanatidou, E., Aravas, N., 2002. Mixed finite element formulations of strain-gradient elasticity problems. Comp. Methods Appl. Mech. Eng. 191, 1723-1751.

Anand, L., Ames, N.M., 2006. On modeling micro-indentation response of an amorphous polymer. Int. J. Plast. 22, 1123-1170.

Argon, A.S., 1973. Theory for the low-temperature plastic deformation of glassy polymers. Phil. Mag. 28, 839-865.

Arinstein A., Burman M., Gendelman, O., Zussman E., 2007. Effect of supramolecular structure on polymer nanofiber elasticity. Nat. Nanotechnol. 2, 59-62.

Arsenlis, A., Parks, D.M., 1999. Crystallographic aspects of geometrically-necessary and statistically-stored dislocation density. Act. Mater. 47, 1597-1611.

Askes, H., Aifantis, E.C., 2002. Numerical modeling of size effects with gradient elasticity - formulation, meshless discretization and examples. Int. J. Frac. 117, 347-358.

Askes, H., Gutierrez, M.A., 2006. Implicit gradient elasticity. Int. J. Num. Meth. Eng. 67, 400-416.

Askes, H., Pamin, J., de Borst, R., 2000. Dispersion analysis and element-free Galerkin solutions of second and fourth-order gradient-enhanced damage models. Int. J. Num. Meth. Eng. 49, 811-832.

Balta Calleja, F.J., Flores, A., Michler, G.H., 2004. Microindentation studies at the near surface of glassy polymers: influence of Molecular weight. J. Appl. Polym. Sci. 93, 1951-1956.

Bardia, P., Narasimhan, R., 2006. Characterisation of pressure-sensitive yielding in polymers. Strain 42, 187-196.

Bittencourt, E., Needleman, A., Gurtin, M.E., van der Giessen, E., 2003. A comparison of nonlocal continuum and discrete dislocation plasticity predictions. J. Mech. Phys. Sol. 51, 281-310.

Briscoe, B.J., Fiori, L., Pelillo, E., 1998. Nanoindentation of polymeric surfaces. J. Phys. D 31, 2395-2405.

Chandrashekar, G., Alisafaei, F., Han, C.-S., 2015. Length scale dependent deformation in natural rubber. J. Appl. Polym. Sci. $132,42683$.

Charitidis, C., 2011. Nanoscale Deformation and Nanomechanical Properties of Polydimethylsiloxane (PDMS). Ind. Eng. Chem. Res. 50, 565-70.

Chong, A.C.M., Lam, D.C.C., 1999. Strain gradient plasticity effect in indentation hardness of polymers. J. Mater. Res. 14, 4103-4110.

Dahlberg, C.F.O., Saito, Y., Öztop, M.S., Kysar, J.W., 2014. Geometrically necessary dislocation density measurements associated with different angles of indentations. Int. J. Plast. 54, 81-95.

de Souza Neto, E.A., Peric, D. Owen, D.R.J., 2008. Computational methods for plasticity: theory and applications. John Wiley $\&$ Sons Ltd.

Doerner, M.F., Nix, W.D., 1986. A method for interpreting the data from depth-sensing indentation instruments. J. Mater. Res. 1, 601-609.

Du Bois, P.A., Kolling, S., Koesters, M., Frank, T., 2006. Material behaviour of polymers under impact loading. Int. J. Imp. Eng. 32, 725-740.

Dunne, F., Petrinic, N., 2005. Introduction to computational plasticity. Oxford University Press, New York.

Dutta, A.K., Penumadu, D., Files, B., 2004. Nanoindentation testing for evaluating modulus and hardness of single-walled carbon nanotube-reinforced epoxy composite. J. Mater. Res. 19, 158-164.

Fischer, P., Klassen, M., Mergheim, J., Steinmann, P., Müller, R., 2011. Isogeometric analysis of 2D gradient elasticity. Comput. Mech. 47, 325-334.

Fleck, N.A., Muller, G.M., Ashby, M.F., Hutchinson, J.W., 1994. Strain gradient plasticity: theory and experiment. Act. Metall. Mater. 42, 475-487.

Garg, N., Han, C.-S., 2013. A penalty finite element approach for couple-stress elasticity. Comput. Mech. 52, 709-720.

Garg, N., Han, C.-S., 2015. An axisymmetric finite element formulation for couple-stress elasticity. Arch. Appl. Mech., 85, 587600. 
Gerberich, W.W., Tymiak, N.I., Grunlan, J.C., Horstemeyer, M.F., Baskes, M.I., 2002. Interpretations of indentation size effects. J. Appl. Mech. 69, 433-442.

Ghosh, S., Kumar, A., Sundararaghavan, V., Waas, A.M., 2013. Non-local modeling of epoxy using an atomistically-informed kernel. Int. J. Sol. Struc. 50, 2837-2845.

Govaert, L.E., Timmermans, P.H.M., Brekelmans, W.A.M., 2000. The influence of intrinsic strain softening on strain localization in polycarbonate: modeling and experimental validation. J. Eng. Mater. Tech. ASME 122, 177-185.

Gudmundson, P., 2006. Modelling of length scale effects in viscoelastic materials. Euro. J. Mech. A/Sol. 25, 379-388.

Han, C.-S., 2010. Influence of the molecular structure on indentation size effect in polymers. Mater. Sci. Eng. A 527, 619-624.

Han, C.-S., Ma, A., Roters, F., Raabe, D., 2007. A Finite Element approach with patch projection for strain gradient plasticity formulations. Int. J. Plast. 23, 690-710.

Han, C.-S., Nikolov, S., 2007. Indentation size effects of polymers and related rotation gradients. J. Mater. Res. 22, 1662-1672.

Han, C.-S., Sanei, S.H.R., Alisafaei, F., 2015. On the origin of indentation size effects and depth dependent elastic moduli in polymers. J. Polym. Eng., in press, DOI: 10.1515/polyeng-2015-0030.

Hard af Segerstad, P., Toll, S., Larsson, R., 2009. A micropolar theory for the finite elasticity of open-cell cellular solids. Proc. R. Soc. A 465, 843-865.

Hertz, H., 1882. Über die Berührung fester elastischer Körper (On the contact of rigid elastic solids). Journal für die Reine und Angewandte Mathematik 29, 156-171.

Hu, Y., Shen, L., Yang, H., Wang, M., Liu, T., Liang, T., Zhang, J., 2006. Nanoindentation studies on Nylon 11/clay nanocomposite. Polym. Test. 25, 492-497.

Huang, Y., Qu, S., Hwang, K.C., Li, M., Gao, H., 2004. A conventional theory of mechanism-based strain gradient plasticity. Int. J. Plast. 20, 753-782.

ISO14577-1, 2002. Metallic materials - Instrumented indentation test for hardness and materials parameters - Part 1: test method. International Organization for Standardization, Geneva, Switzerland.

Jatin, Sudarkodi, V., Basu, S., 2014. Investigations into the origins of plastic flow and strain hardening in amorphous glassy polymers. Int. J. Plast. 56, 139-155.

Koumoulos, E.P., Jagdale, P., Kartsonakis, I.A., Giorcelli, M., Tagliaferro, A., Charitidis, C.A., 2015. Carbon nanotube/polymer nanocomposites: A study on mechanical integrity through nanoindentation. Polym. Compos. 36, 1432-1446.

Krairi, A., Doghri, I., 2014. A thermodynamically-based constitutive model for thermoplastic polymers coupling viscoelasticity, viscoplasticity and ductile damage. Int. J. Plast. 60, 163-181.

Lakes, R.S., 1986. Experimental microelasticity of two porous solids. Int. J. Sol. Struc. 22, 55-63.

Lakes, R.S., 1991. Experimental micro mechanics methods for conventional and negative Poisson's ratio cellular solids as Cosserat continua. Trans. ASME 113, 148-155.

Lam, D.C.C., Chong, A.C.M., 1999. Indentation model and strain gradient plasticity law for glassy polymers. J. Mater. Res. 14, 3784-3788.

Lam, D.C.C., Chong, A.C.M., 2000. Effect of cross-link density on strain gradient plasticity in epoxy. Mater. Sci. Eng. A 281, 156-161.

Lam, D.C.C., Chong, A.C.M., 2001. Characterization and modeling of specific strain gradient modulus of epoxy. J. Mater. Res. $16,558-563$.

Lam, D.C.C., Yang, F., Chong, A.C.M., Wang, J., Tong, P., 2003. Experiments and theory in strain gradient elasticity. J. Mech. Phys. Sol. 51, 1477-1508.

Lee, M.-G., Han, C.-S., 2012. An explicit finite element approach for strain gradient plasticity formulations. Comput. Mech. 49, 171-183.

Li, X., Tang, H., 2005. A consistent return mapping algorithm for pressure-dependent elastoplastic Cosserat continua and modelling of strain localisation. Comput. Struct. 83, 1-10.

Lim, Y.Y., Chaudhri, M.M., 2006. Indentation of elastic solids with a rigid Vickers pyramidal indenter. Mech. Mater. 38, 12131228. 
Liu, D., He, Y., Dunstan, D., Zhang, B., Gan, Z., Hu, P., Ding, H., 2013. Toward a further understanding of size effects in the torsion of thin metal wires: An experimental and theoretical assessment. Int. J. Plast. 41, 30-52.

Liu, Y., Truss, R.W., 1994. A study of tensile yielding of isotactic polypropylene. J. Polym. Sci. B-Polym. Phys. 32, $2037-2047$.

Lu, Y.C., Jones, D.C., Tandon, G.P., Putthanarat, S., Schoeppner, G.A., 2010. High temperature nanoindentation of PMR-15 polyimide. Exp. Mech. 50, 491-499.

Lyu, H., Ruimi, A., Zbib, H.M., 2015. A dislocation-based model for deformation and size effect in multi-phase steels. Int. J. Plast. 72, 44-59.

Ma, Q., Clarke, D.R., 1995. Size dependent hardness of silver single crystals. J. Mater. Res. 10, 853-863.

McDowell, D.L., 2010. A perspective on trends in multiscale plasticity. Int. J. Plast. 26, 1280-1309.

McFarland, A.W., Colton, J.S., 2005. Role of material microstructure in plate stiffness with relevance to microcantilever sensors. J. Micromech. Microeng. 15, 1060-1067.

Mora, R.J., Waas, A.M., 2002. Strength scaling of brittle graphitic foam. Proc. Roy. Soc. A-Math. Phys. Eng. Sci. 458, 16951718.

Mora, R.J., Waas, A.M., 2007. Evaluation of the Micropolar elasticity constants for honeycombs. Act. Mech. 192, 1-16.

Motz, C., Schöberl, T., Pippan, R., 2005. Mechanical properties of micro-sized copper bending beams machined by the focused ion beam technique. Act. Mater. 53, 4269-4279.

Nikolov, S., Han, C.-S., Raabe, D., 2007. On the origin of size effects in small-strain elasticity of solid polymers. Int. J. Sol. Struc. 44, 1582-1592.

Nix, W.D., Gao, H., 1998. Indentation size effects in crystalline materials: A law for strain gradient plasticity. J. Mech. Phys. Sol. 46, 411-425.

Oliver, W.C., Pharr, G.M., 1992. Improved technique for determining hardness and elastic modulus using load and displacement sensing indentation experiments. J. Mater. Res. 7, 1564-1583.

Oyen, M.L., Cook, R.F., 2003. Load-displacement behavior during sharp indentation of viscous-elastic-plastic materials. J. Mater. Res. 18, 139-150.

Papanicolopulos, S.-A., Zervos, A., Vardoulakis, I., 2009. A three-dimensional $C^{1}$ finite element for gradient elasticity. Int. J. Numer. Methods Eng. 77, 1396-1415.

Petera, J., Pittman, J.F.T., 1994. Isoparametric hermite elements. Int. J. Numer. Methods Eng. 37, 3489-3519.

Poulain, X., Benzerga, A.A., Goldberg, R.K., 2014. Finite-strain elasto-viscoplastic behavior of an epoxy resin: Experiments and modeling in the glassy regime. Int. J. Plast. 62, 138-161.

Raghava, R., Caddell, R.M., 1973. The macroscopic yield behaviour of polymers. J. Mater. Sci. 8, 225-232.

Richeton, J., Ahzi, S., Daridon, L., Remond, Y., 2005. A formulation of the cooperative model for the yield stress of amorphous polymers for a wide range of strain rates and temperatures. Polym. 46, 6035-6043.

Rodriguez, R., Gutierrez, I, 2003. Correlation between nanoindentation and tensile properties influence of the indentation size effect. Mater. Sci. Eng. A 361, 377-384.

Rottler, J., Robbins, M.O., 2001. Yield conditions for deformation of amorphous polymer glasses. Phys. Rev. E 64, 051801 Part: 1.

Saha, R., Nix, W.D., 2002. Effects of the substrate on the determination of thin film mechanical properties by nanoindentation. Act. Mater. 50, 23-38.

Sanchez, M., Rams, J., Campo, M., Jimenez-Suarez, A., Urena, A., 2011. Characterization of carbon nanofiber/epoxy nanocomposites by the nanoindentation technique. Compos. B 42, 638-644.

Seltzer, R., Cisilino, A.P., Frontini, P.M., Mai, Y.-W., 2011. Determination of the Drucker-Prager parameters of polymers exhibiting pressure-sensitive plastic behaviour by depth-sensing indentation. Int. J. Mech. Sci. 53, 471-478.

Seltzer, R., Kim, J.-K., Mai, Y.-W., 2011. Elevated temperature nanoindentation behaviour of polyamide 6. Polym. Int. 60, 1753-1761.

Shen, L., Liu, T., Lv, P., 2005. Polishing effect on nanoindentation behavior of nylon 66 and its nanocomposites. Polym. Test. 24, 746-749.

Shu, J.Y., Fleck, N.A., 1998. The prediction of a size effect in micro-indentation. Int. J. Solids Struct. 35, 1363-1383. 
Shu, J.Y., King, W.E., Fleck, N.A., 1999. Finite elements for materials with strain gradient effects. Int. J. Num. Meth. Eng. 44, 373-391.

Simo, J.C., Hughes, T.J.R., 1998. Computational inelasticity. Springer, Berlin.

Sperling, L.H., 1993. Introduction to physical polymer science. John Wiley \& Sons.

Srinivasa, A.R., Reddy, J.N., 2013. A model for a constrained, finitely deforming, elastic solid with rotation gradient dependent strain energy, and its specialization to von Karman plates and beams. J. Mech. Phys. Sol. 61, 873-885.

Sun, L., Han, R.P.S, Wang, J., Lim, C.T., 2008. Modeling the size-dependent elastic properties of polymeric nanofibers. Nanotech. 19, 455706.

Swaddiwudhipong, S., Poh, L.H., Hua, J., Liu, Z.S., Tho, K.K., 2005. Modeling nano-indentation tests of glassy polymers using finite elements with strain gradient plasticity. Mater. Sci. Eng. A 404, 179-187.

Swadener, J.G., George, E.P., Pharr, G.M., 2002. The correlation of the indentation size effect measured with indenters of various shapes. J. Mech. Phys. Sol. 50, 681-694.

Szabo, L., Kossa, A., 2012. A new exact integration method for the Drucker-Prager elastoplastic model with linear isotropic hardening. Int. J. Solids Struct. 49, 170-190.

Taheri-Nassaj, N., Zbib, H.M., 2015. On dislocation pileups and stress-gradient dependent plastic flow. Int. J. Plast. 74, 1-16.

Tang, Z., Shen, S., Atluri, S.N., 2003. Analysis of materials with strain-gradient effects: a meshless local Petrov Galerkin (MLPG) approach, with nodal displacements only. Comput. Models Eng. Sci. 4, 177-196.

Tatiraju, R.V.S., Han, C.-S., 2010. Rate dependence of indentation size effects in filled silicone rubber. J. Mech. Mater. Struc. 5, $277-288$.

Tjernlund, J.A., Gamstedt, E.K., Xu, Z.-H., 2004. Influence of molecular weight on strain-gradient yielding in polystyrene. Polym. Eng. Sci. 44, 1987-1997.

Truss, R.W., Clarke, P.L., Duckett, R.A., Ward, I.M., 1984. The dependence of yield behavior on temperature, pressure, and strain rate for linear polyethylenes of different molecular-weight and morphology. J. Polym. Sci. B - Polym. Phys. 22, 191209.

Tymiak, N.I., Kramer, D.E., Bahr, D.F., Wyrobek, T.J., Gerberich, W.W., 2001. Plastic strain and strain gradients at very small indentation depths. Act. Mater. 49, 1021-1034.

Voyiadjis, G.Z., Shojaei, A., Mozaffari, N., 2014. Strain gradient plasticity for amorphous and crystalline polymers with application to micro- and nano-scale deformation analysis. Polym. 55, 4182-4198.

Ward, I.M., Sweeney, J., 2013. Mechanical properties of solid polymers. 3rd Edition, John Wiley \& Sons.

Wells, G.N., Garikipati, K., Molari, L., 2004. A discontinuous Galerkin formulation for a strain gradient-dependent damage model. Comp. Methods Appl. Mech. Eng. 193, 3633-3645.

Wrucke, A.J., Han, C.-S., Majumdar, P., 2013. Indentation size effect of multiple orders of magnitude in polydimethylsiloxane. J. Appl. Polym. Sci. 128, 258-264.

Xia, Z.C., Hutchinson, J.W., 1996. Crack tip fields in strain gradient plasticity. J. Mech. Phys. Solids 44, 1621-1648.

Xu, W.H., Xiao, Z.Y, Zhang, T.Y., 2005. Mechanical properties of silicone elastomer on temperature in biomaterial application. Mater. Lett. 59, 2153-2155.

Yang, F., Chong, A.C.M., Lam, D.C.C., Tong, P., 2002. Couple stress based strain gradient theory for elasticity. Int. J. Sol. Struc. 30, 2731-2743.

Zervos, A., Papanastasiou, P., Vardoulakis, I., 2001. A finite element displacement formulation for gradient elastoplasticity. Int. J. Num. Meth. Eng. 50, 1369-1388.

Zervos, A., Papanastasiou, P., Vardoulakis, I., 2001. A finite element displacement formulation for gradient elastoplasticity. Int. J. Numer. Methods Eng. 50, 1369-1388.

Zhai, M., McKenna, G.B., 2014. Viscoelastic modeling of nanoindentation experiments: a multicurve method. J. Polym. Sci. B: Polym. Phys. 52, 633-639.

Zhang, T.-Y., Xu, W.-H., 2002. Surface effects on nanoindentation. J. Mater. Res. 17, 1715-1720.

Zhang, T.Y., Xu, W.H., Zhao, M.H., 2004. The role of plastic deformation of rough surfaces in the size-dependent hardness. Act. Mater. 52, 57-68. 


\section{Figure captions:}

1. (a) Size effects observed in elastic bending of epoxy micro-beams where the normalized bending stiffness (rigidity) increases with decreasing beam thickness. The normalized bending stiffness is determined as $D^{\prime} / D^{\prime}{ }_{o}$ where $D^{\prime}=Q a^{3} /\left(3 w_{0} h^{3}\right)$ is the bending stiffness (rigidity), $D^{\prime}{ }_{o}$ is the bending stiffness predicted by the classical elasticity theory $\left(D^{\prime}\right.$ at large thickness where the effect of higher order displacement gradients can be neglected), $Q$ is the vertical loading force applied on the right end of the beam (see Fig. 1(b)), $a$ is the length of the beam, $h$ is the thickness of the beam, and $w_{0}$ is the maximum vertical displacement of the beam. The length to thickness ratio $a / h$ was kept constant at 10).

(b) Experimental loading-unloading curve of epoxy cantilever beam illustrating elastic behavior (redrawn from Lam et al., 2003).

2. Indentation size effect in aluminum: indentation hardness $H_{\mathrm{I}}$ (as defined in ISO14577-1, 2002) versus indentation depth $h_{\max }$ (indentation depth at maximum applied force $F_{\max }$ before holding time).

3. Indentation size effect in epoxy: indentation hardness $H_{\mathrm{I}}$ versus indentation depth $h_{\text {max }}$ obtained with $20 \mathrm{~s}$ loading, holding and unloading times (Present).

4. Indentation size effect in epoxy: universal hardness $H_{\mathrm{U}}$ versus indentation depth $h_{\text {max }}$ obtained with 20 s loading time.

5. Schematic representation of load $F$ versus indenter displacement $h$ for an indentation experiment.

6. Elastic moduli of aluminum obtained according to Oliver \& Pharr (1992) with a Berkovich indenter tip, $E_{\mathrm{B}}^{\mathrm{O} \& \mathrm{P}}$, versus indentation depth $h_{\text {max }}$.

7. Elastic moduli of epoxy determined with a Berkovich indenter tip $E_{\mathrm{B}}^{\mathrm{O} \& \mathrm{P}}$ (according to O\&P's method with $20 \mathrm{~s}$ loading, holding, and unloading times), a spherical indenter tip $E_{\text {sphere }}^{\text {O\&P }}$ and $E_{\text {sphere }}^{\mathrm{Hertz}}$ (according to O\&P's method and Hertz theory with $20 \mathrm{~s}$ loading time, $20 \mathrm{~s}$ unloading time, and no holding time).

8. Second order gradients in the displacements $\chi$ for different tip geometries and their dependence on $h_{\max }$.

9. Polystyrene (PS): Normalized values of indentation hardness, $\eta_{\mathrm{I}}=H_{\mathrm{I}} / H_{\mathrm{I}}^{(\mathrm{M})}$, elastic modulus according to Oliver \& Pharr (1992), $\lambda_{\mathrm{B}}^{\mathrm{O} \& \mathrm{P}}=E_{\mathrm{B}}^{\mathrm{O} \& \mathrm{P}} / E_{\mathrm{B}}^{\mathrm{O} \& \mathrm{P}(\mathrm{M})}$, and universal hardness, $\eta_{\mathrm{U}}=H_{\mathrm{U}} / H_{\mathrm{U}}^{(\mathrm{M})}$, with $10 \mathrm{~s}$ loading, holding and unloading times (Briscoe et al., 1998). Normalized universal hardness, $\eta_{\mathrm{U}}$, and elastic modulus according to Doerner \& Nix (1986), $\lambda_{\mathrm{B}}^{\mathrm{D} \& \mathrm{~N}}=E_{\mathrm{B}}^{\mathrm{D} \& \mathrm{~N}} / E_{\mathrm{B}}^{\mathrm{D} \& \mathrm{~N}(\mathrm{M})}$, with constant tip velocity during loading and unloading together with $6 \mathrm{~s}$ holding time (Balta Calleja et al., 2004) superscript $(\mathrm{M})$ indicates macroscopic values.

10. Polycarbonate (PC): Normalized values of indentation hardness, $\eta_{\mathrm{I}}=H_{\mathrm{I}} / H_{\mathrm{I}}^{(\mathrm{M})}$, elastic modulus according to Oliver \& Pharr (1992), $\lambda_{\mathrm{B}}^{\mathrm{O} \& \mathrm{P}}=E_{\mathrm{B}}^{\mathrm{O} \& \mathrm{P}} / E_{\mathrm{B}}^{\mathrm{O} \& \mathrm{P}(\mathrm{M})}$, and universal hardness, $\eta_{\mathrm{U}}=H_{\mathrm{U}} / H_{\mathrm{U}}^{(\mathrm{M})}$, with $10 \mathrm{~s}$ loading, holding and unloading times (Briscoe et al., 1998) - superscript (M) indicates macroscopic values. 
11. Poly methyl methacrylate (PMMA): Normalized values of indentation hardness, $\eta_{\mathrm{I}}=H_{\mathrm{I}} / H_{\mathrm{I}}^{(\mathrm{M})}$, elastic modulus according to Oliver \& Pharr (1992), $\lambda_{\mathrm{B}}^{\mathrm{O} \& \mathrm{P}}=E_{\mathrm{B}}^{\mathrm{O} \& \mathrm{P}} / E_{\mathrm{B}}^{\mathrm{O} \& \mathrm{P}(\mathrm{M})}$, and universal hardness, $\eta_{\mathrm{U}}=H_{\mathrm{U}} / H_{\mathrm{U}}^{(\mathrm{M})}$, with $10 \mathrm{~s}$ loading, holding and unloading times (Briscoe et al., 1998) - superscript (M) indicates macroscopic values.

12. Degrees of freedom for 8-node element, full and reduced integration points are represented by $\mathbf{x}$ and O, respectively.

13. Normalized force versus $h_{\max }$ obtained from the wedge indentation simulations. Normalized force is calculated as the ratio of $F_{\mathrm{o}} / F_{*}$ where $F_{\mathrm{o}}$ is the reaction force when the effects of rotation gradients are taken into account (present formulation) and $F_{*}$ is the reaction force without the presence of rotation gradients (classical elasto-plasticity).

14. Effective stress in GPa (a) without, and (b) with rotation gradients for $100 \mathrm{~nm}$ indentation of epoxy sample with length and thickness of 7 and $2.25 \mu \mathrm{m}$, respectively.

15. Rotation gradients (a) $\chi_{13}$, and (b) $\chi_{23}$ for $100 \mathrm{~nm}$ indentation of epoxy sample with length and thickness of 7 and $2.25 \mu \mathrm{m}$, respectively. 
Fig. 1 (a)

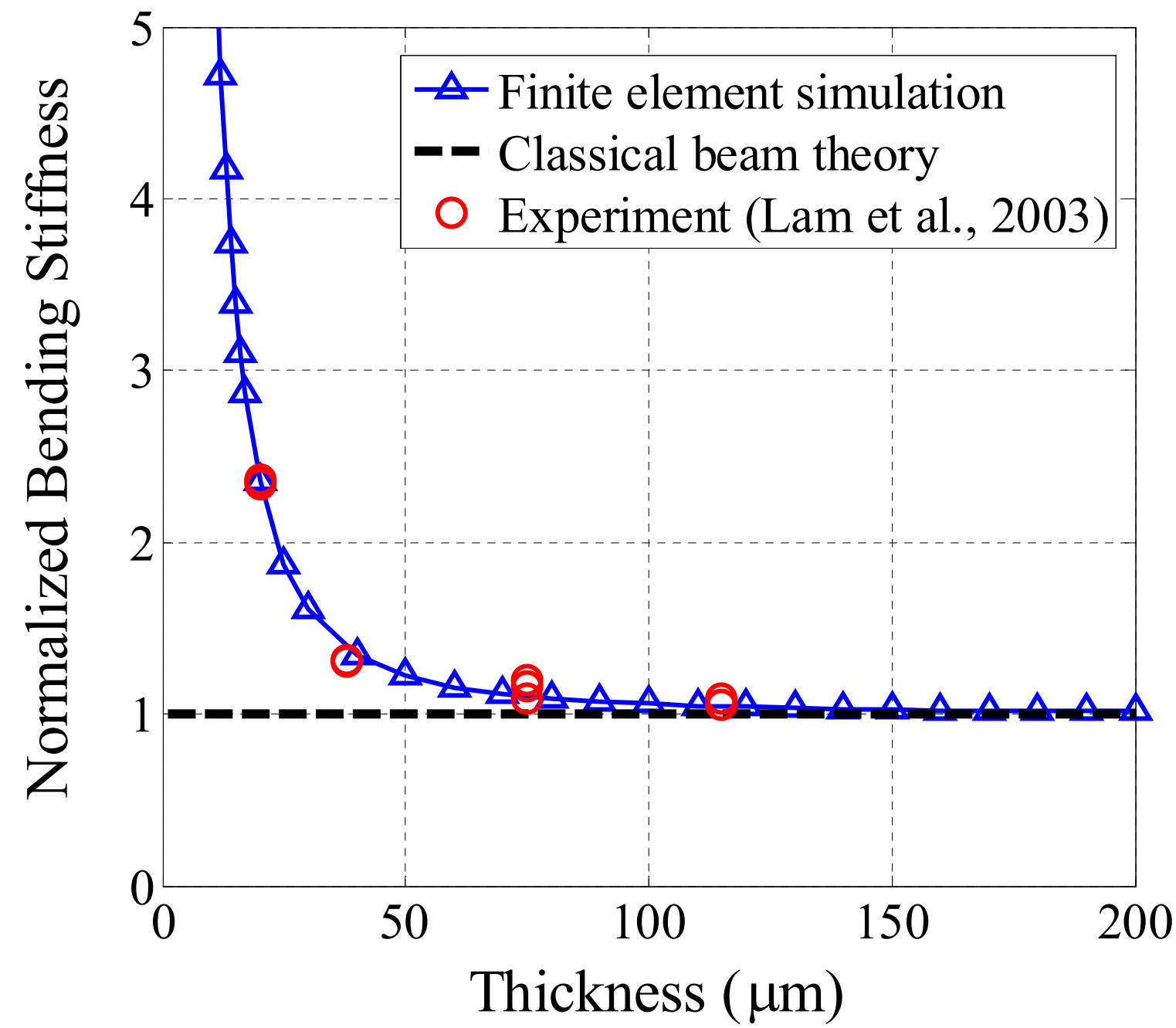

Size effects observed in elastic bending of epoxy micro-beams where the normalized bending stiffness (rigidity) increases with decreasing beam thickness. The normalized bending stiffness is determined as $D^{\prime} / D^{\prime}{ }_{o}$ where $D^{\prime}=Q a^{3} /\left(3 w_{0} h^{3}\right)$ is the bending stiffness (rigidity), $D^{\prime}{ }_{o}$ is the bending stiffness predicted by the classical elasticity theory $\left(D^{\prime}\right.$ at large thickness where the effect of higher order displacement gradients can be neglected), $Q$ is the vertical loading force applied on the right end of the beam (see Fig. 1(b)), $a$ is the length of the beam, $h$ is the thickness of the beam, and $w_{0}$ is the maximum vertical displacement of the beam. The length to thickness ratio $a / h$ was kept constant at 10 ). 
Fig. 1 (b)

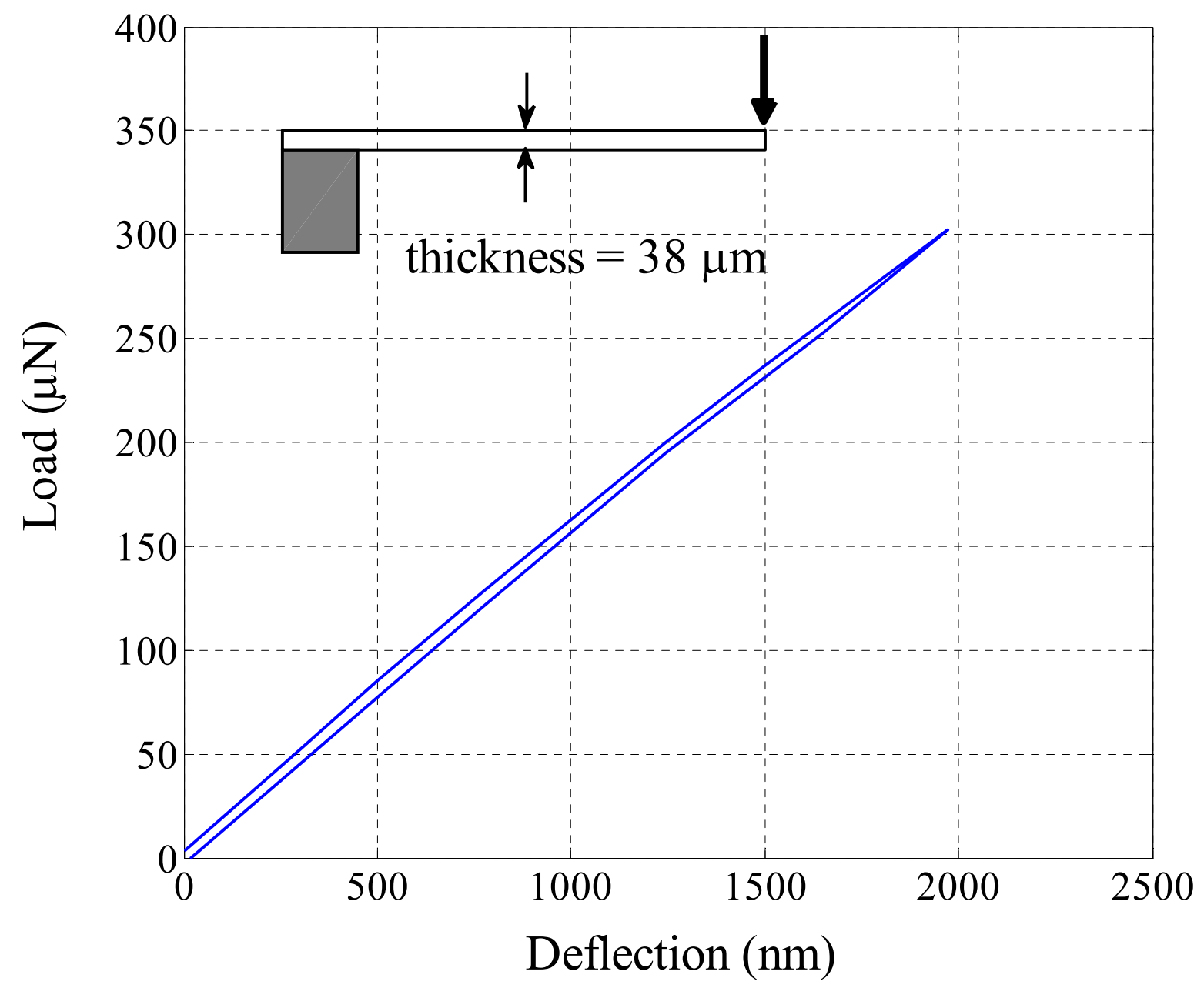

Experimental loading-unloading curve of epoxy cantilever beam illustrating elastic behavior (redrawn from Lam et al., 2003). 
Fig. 2

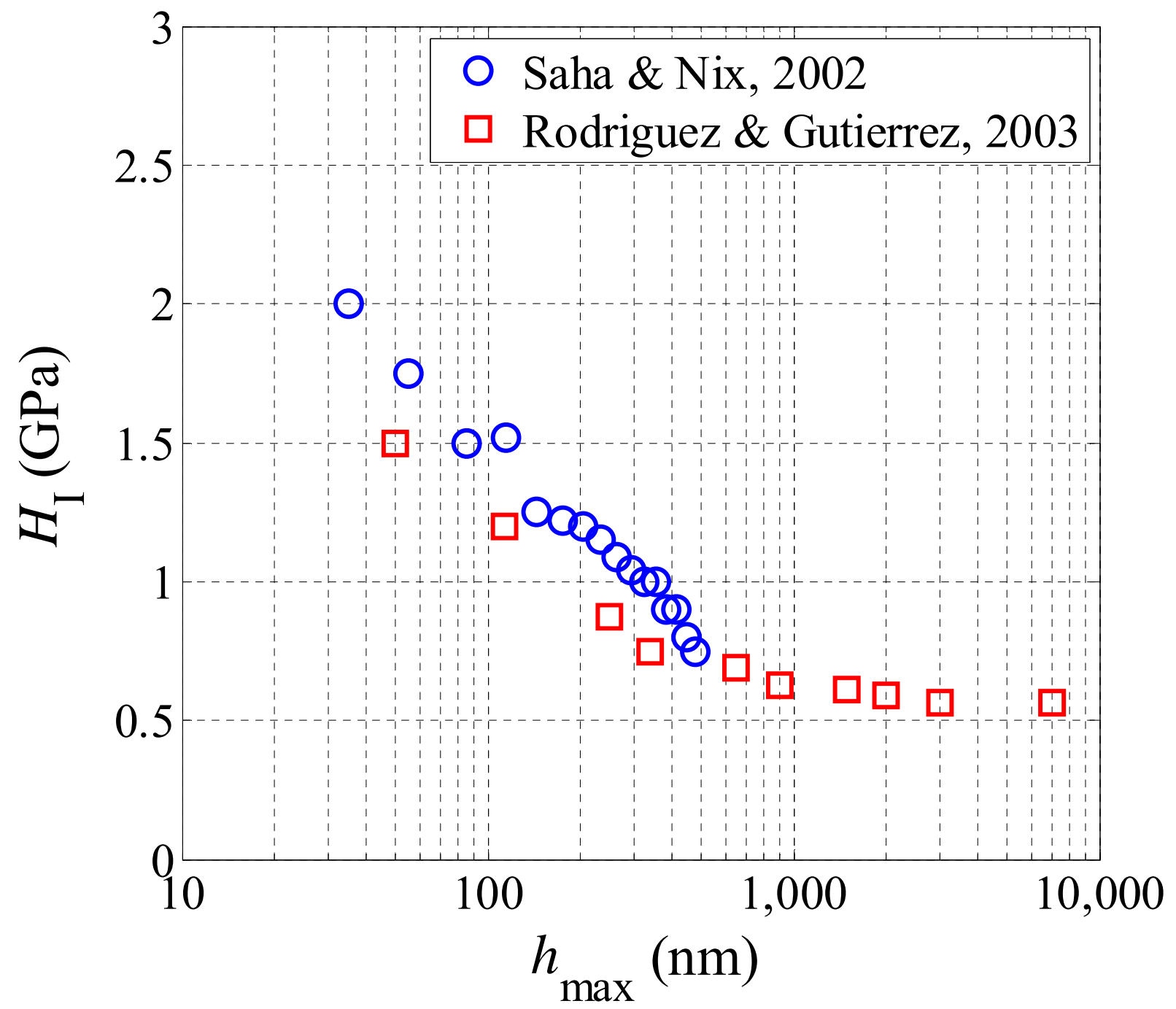

Indentation size effect in aluminum: indentation hardness $H_{\mathrm{I}}$ (as defined in ISO14577-1, 2002) versus indentation depth $h_{\max }$ (indentation depth at maximum applied force $F_{\max }$ before holding time). 
Fig. 3

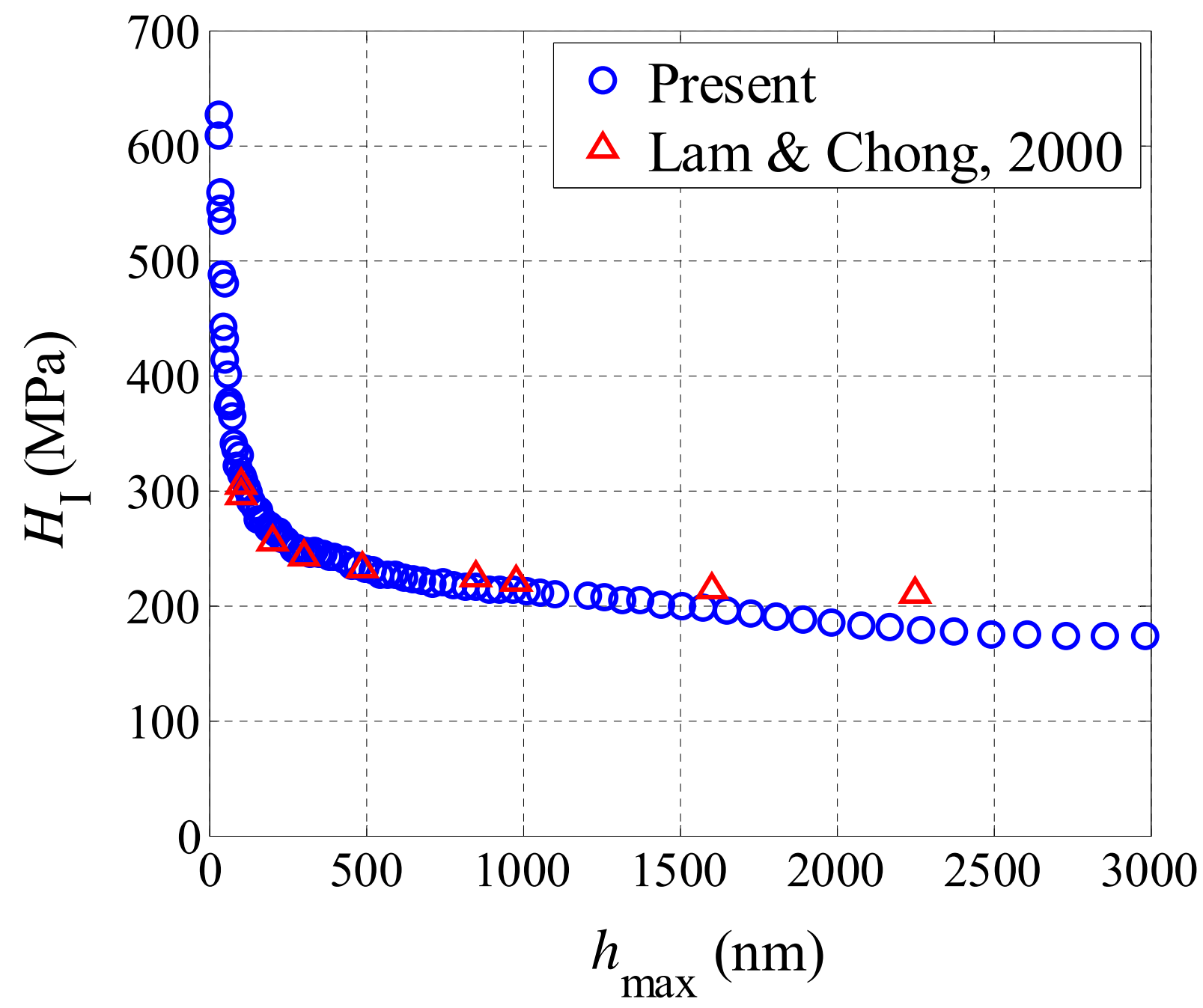

Indentation size effect in epoxy: indentation hardness $H_{\mathrm{I}}$ versus indentation depth $h_{\text {max }}$ obtained with 20 s loading, holding and unloading times (Present) compared with those in (Lam \& Chong, 2000). 
Fig. 4

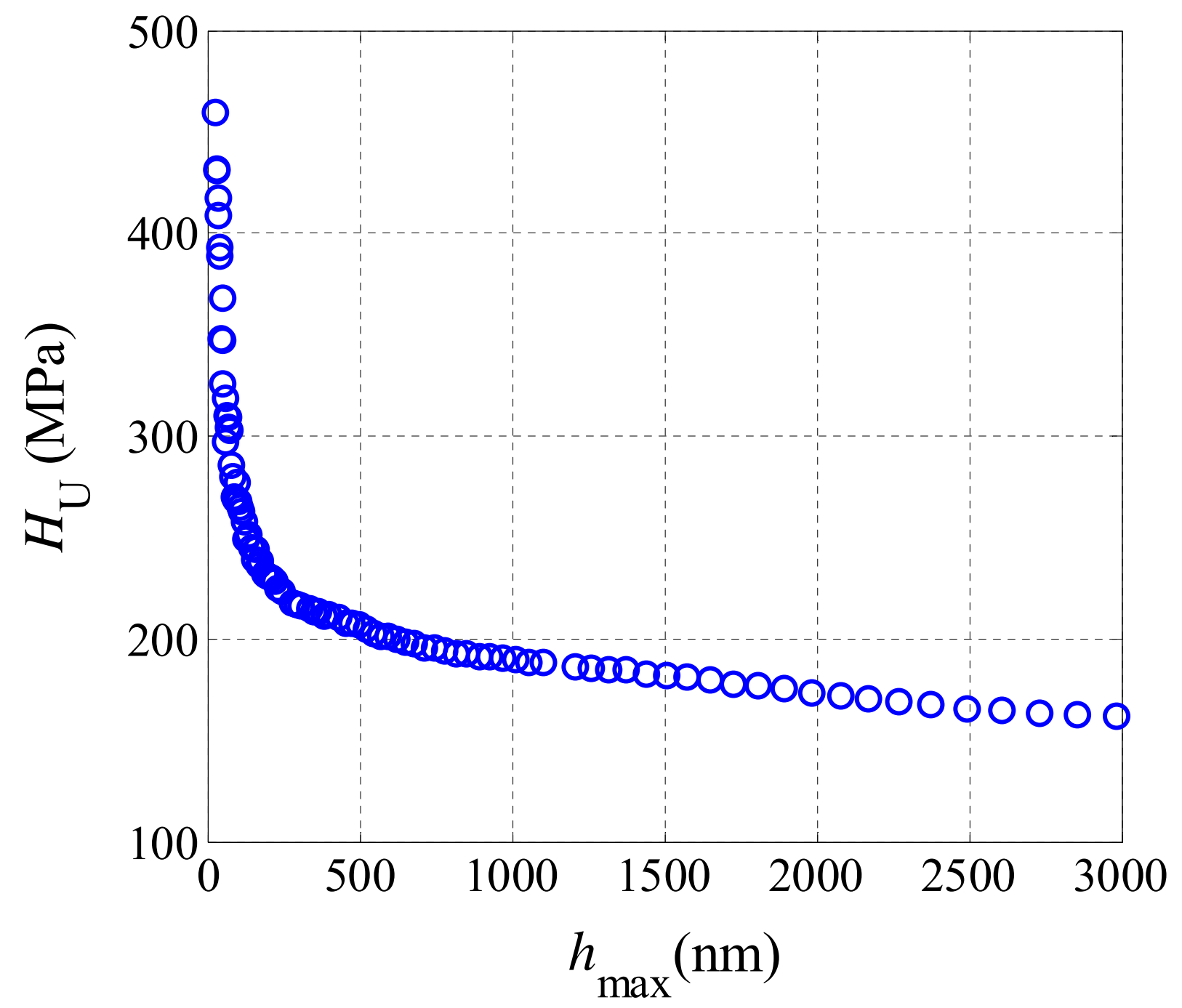

Indentation size effect in epoxy: universal hardness $H_{\mathrm{U}}$ versus indentation depth $h_{\text {max }}$ obtained with $20 \mathrm{~s}$ loading time. 
Fig. 5

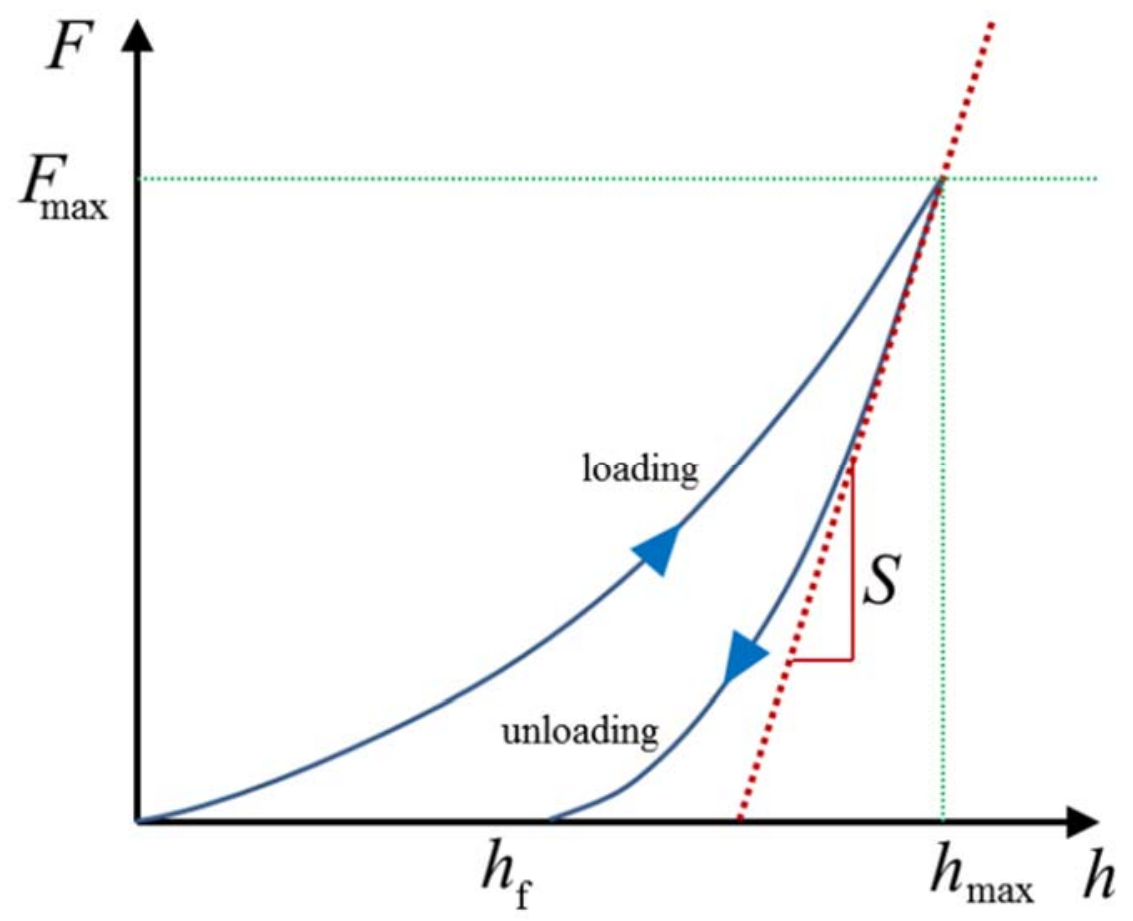

Schematic representation of load $F$ versus indenter displacement $h$ for an indentation experiment. 
Fig. 6

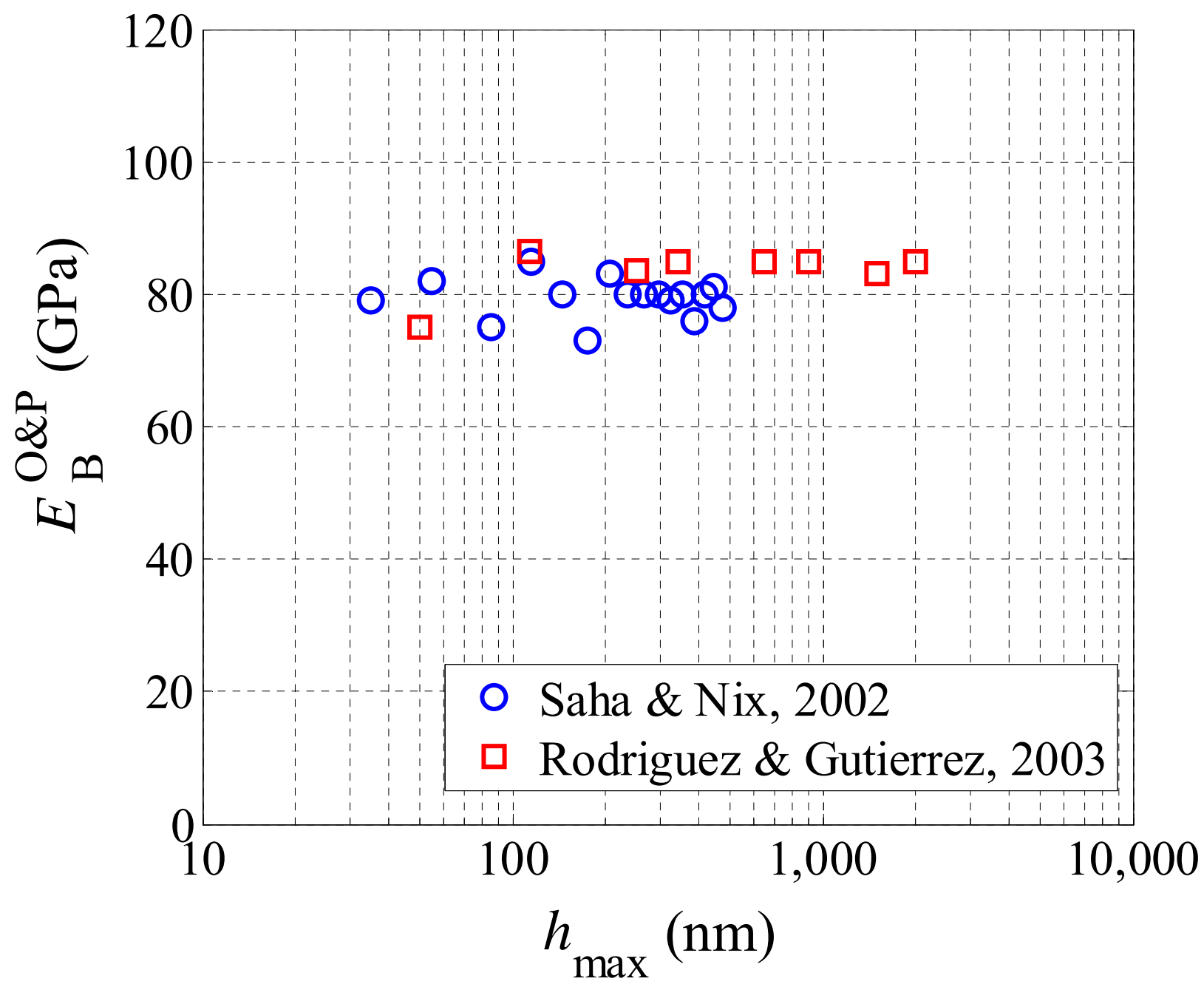

Elastic moduli of aluminum obtained according to Oliver \& Pharr (1992) with a Berkovich indenter tip, $E_{\mathrm{B}}^{\mathrm{O} \& \mathrm{P}}$, versus indentation depth $h_{\text {max }}$. 
Fig. 7

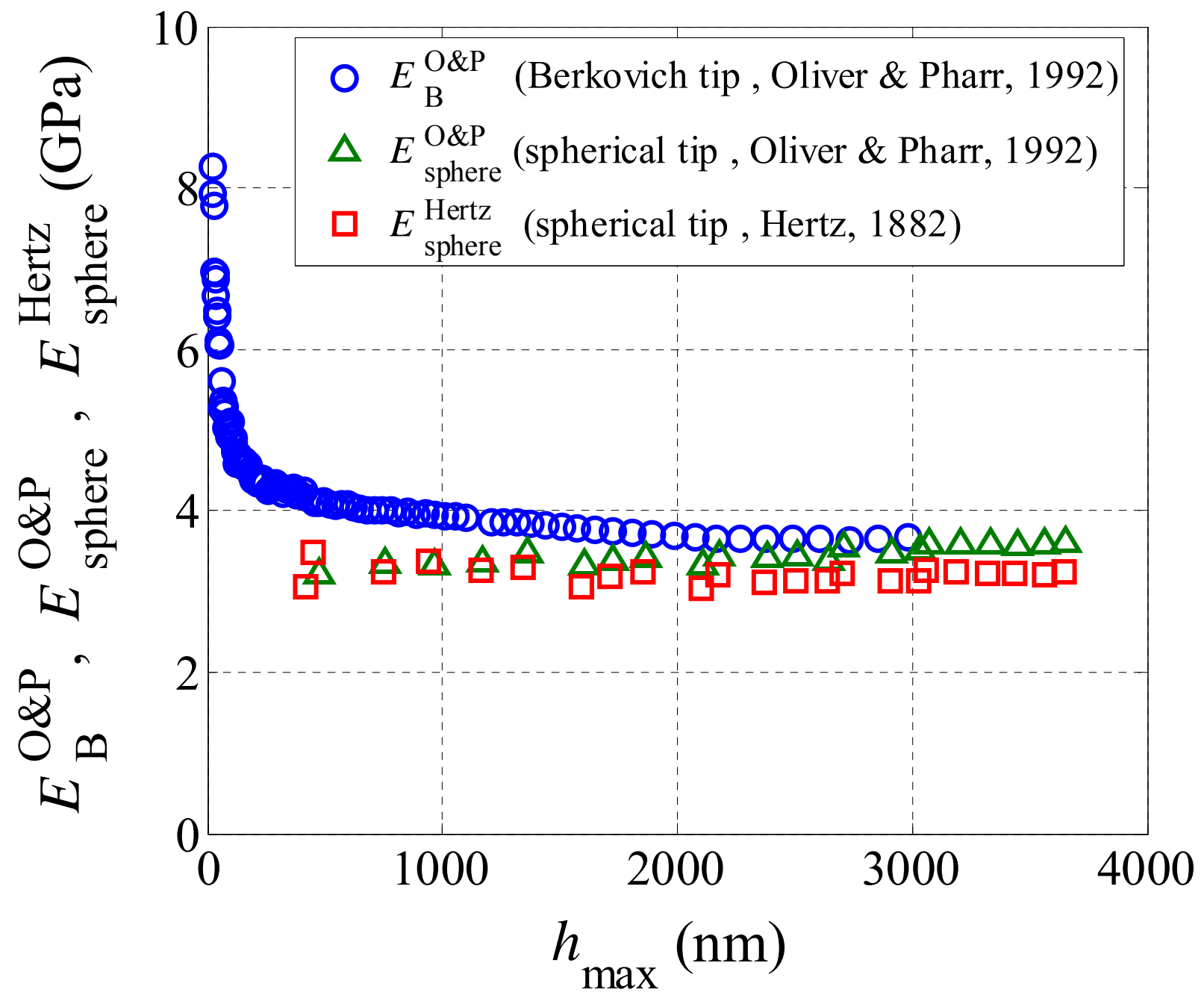

Elastic moduli of epoxy determined with a Berkovich indenter tip $E_{\mathrm{B}}^{\mathrm{O} \& \mathrm{P}}$ (according to O\&P's method with $20 \mathrm{~s}$ loading, holding, and unloading times), a spherical indenter tip $E_{\text {sphere }}^{\mathrm{O} P \mathrm{P}}$ and $E_{\text {sphere }}^{\mathrm{Hertz}}$ (according to O\&P's method and Hertz theory with $20 \mathrm{~s}$ loading time, $20 \mathrm{~s}$ unloading time, and no holding time). 
Fig. 8

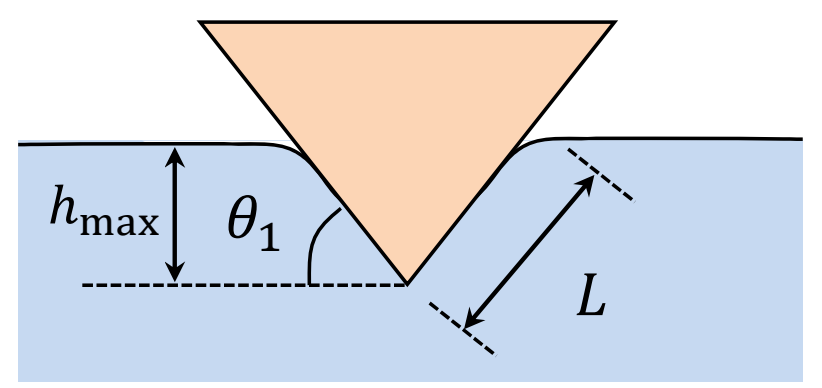

$$
\chi=\frac{\theta_{1}}{L} \propto \frac{1}{h_{\max }}
$$

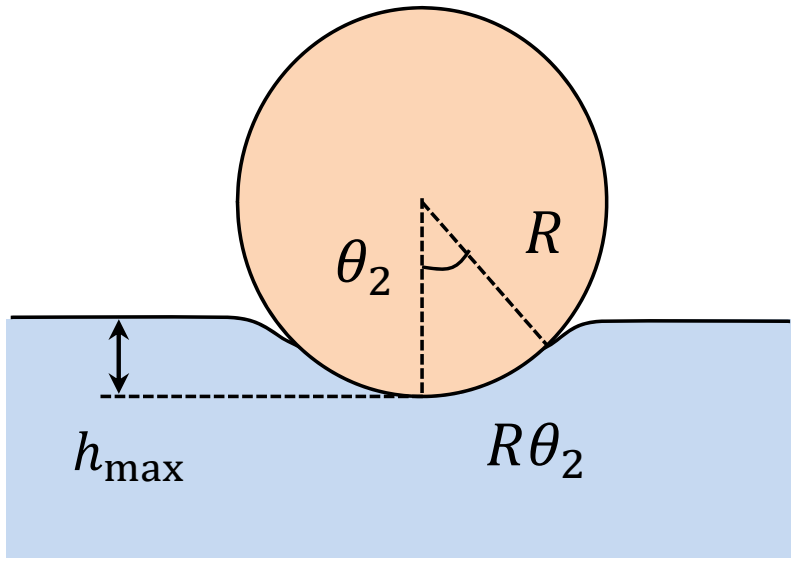

$$
\chi=\frac{\theta_{2}}{R \theta_{2}}=\frac{1}{R}
$$

Second order gradients in the displacements $\chi$ for different tip geometries and their dependence on $h_{\max }$. 
Fig. 9

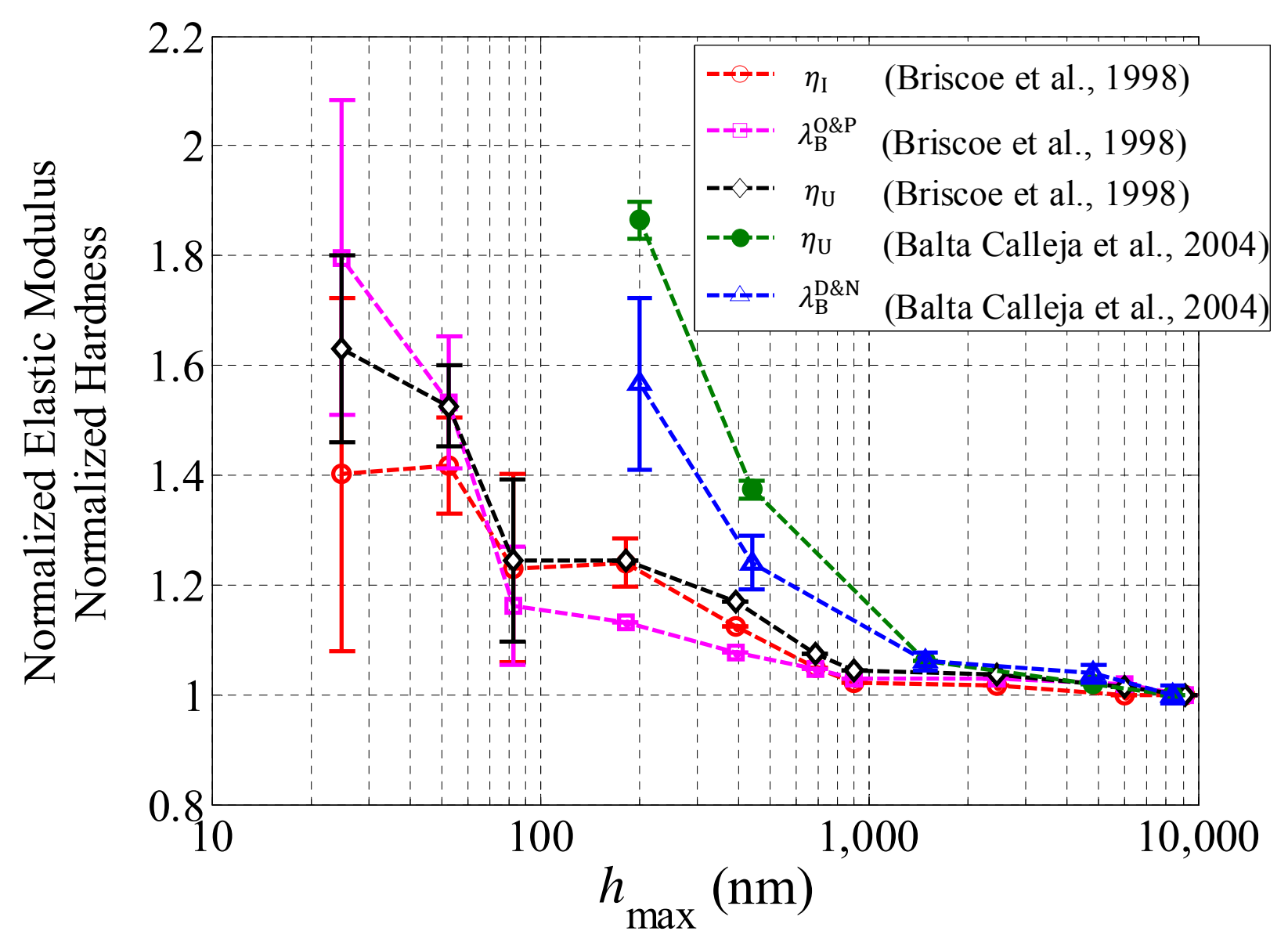

Polystyrene (PS): Normalized values of indentation hardness, $\eta_{\mathrm{I}}=H_{\mathrm{I}} / H_{\mathrm{I}}^{(\mathrm{M})}$, elastic modulus according to Oliver \& Pharr (1992), $\lambda_{\mathrm{B}}^{\mathrm{O} \& \mathrm{P}}=E_{\mathrm{B}}^{\mathrm{O} \& \mathrm{P}} / E_{\mathrm{B}}^{\mathrm{O} \& \mathrm{P}(\mathrm{M})}$, and universal hardness, $\eta_{\mathrm{U}}=H_{\mathrm{U}} / H_{\mathrm{U}}^{(\mathrm{M})}$, with $10 \mathrm{~s}$ loading, holding and unloading times (Briscoe et al., 1998). Normalized universal hardness, $\eta_{\mathrm{U}}$, and elastic modulus according to Doerner \& Nix (1986), $\lambda_{\mathrm{B}}^{\mathrm{D} \& \mathrm{~N}}=E_{\mathrm{B}}^{\mathrm{D} \& \mathrm{~N}} / E_{\mathrm{B}}^{\mathrm{D} \& \mathrm{~N}(\mathrm{M})}$, with constant tip velocity during loading and unloading together with 6 s holding time (Balta Calleja et al., 2004) - superscript (M) indicates macroscopic values. 
Fig. 10

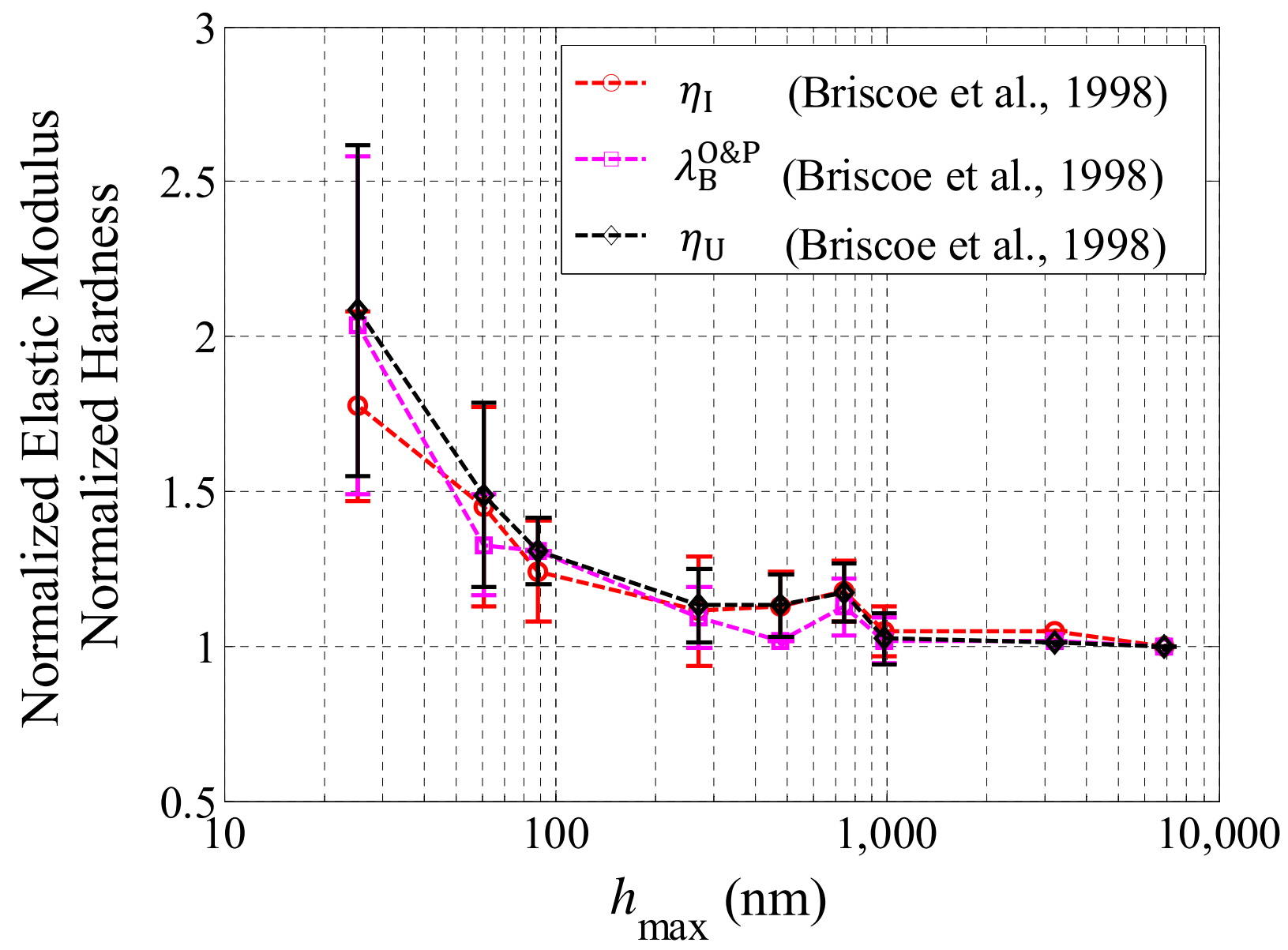

Polycarbonate (PC): Normalized values of indentation hardness, $\eta_{\mathrm{I}}=H_{\mathrm{I}} / H_{\mathrm{I}}^{(\mathrm{M})}$, elastic modulus according to Oliver \& Pharr (1992), $\lambda_{\mathrm{B}}^{\mathrm{O} \& \mathrm{P}}=E_{\mathrm{B}}^{\mathrm{O} \& \mathrm{P}} / E_{\mathrm{B}}^{\mathrm{O} \& \mathrm{P}(\mathrm{M})}$, and universal hardness, $\eta_{\mathrm{U}}=H_{\mathrm{U}} / H_{\mathrm{U}}^{(\mathrm{M})}$, with $10 \mathrm{~s}$ loading, holding and unloading times (Briscoe et al., 1998) - superscript (M) indicates macroscopic values. 
Fig. 11

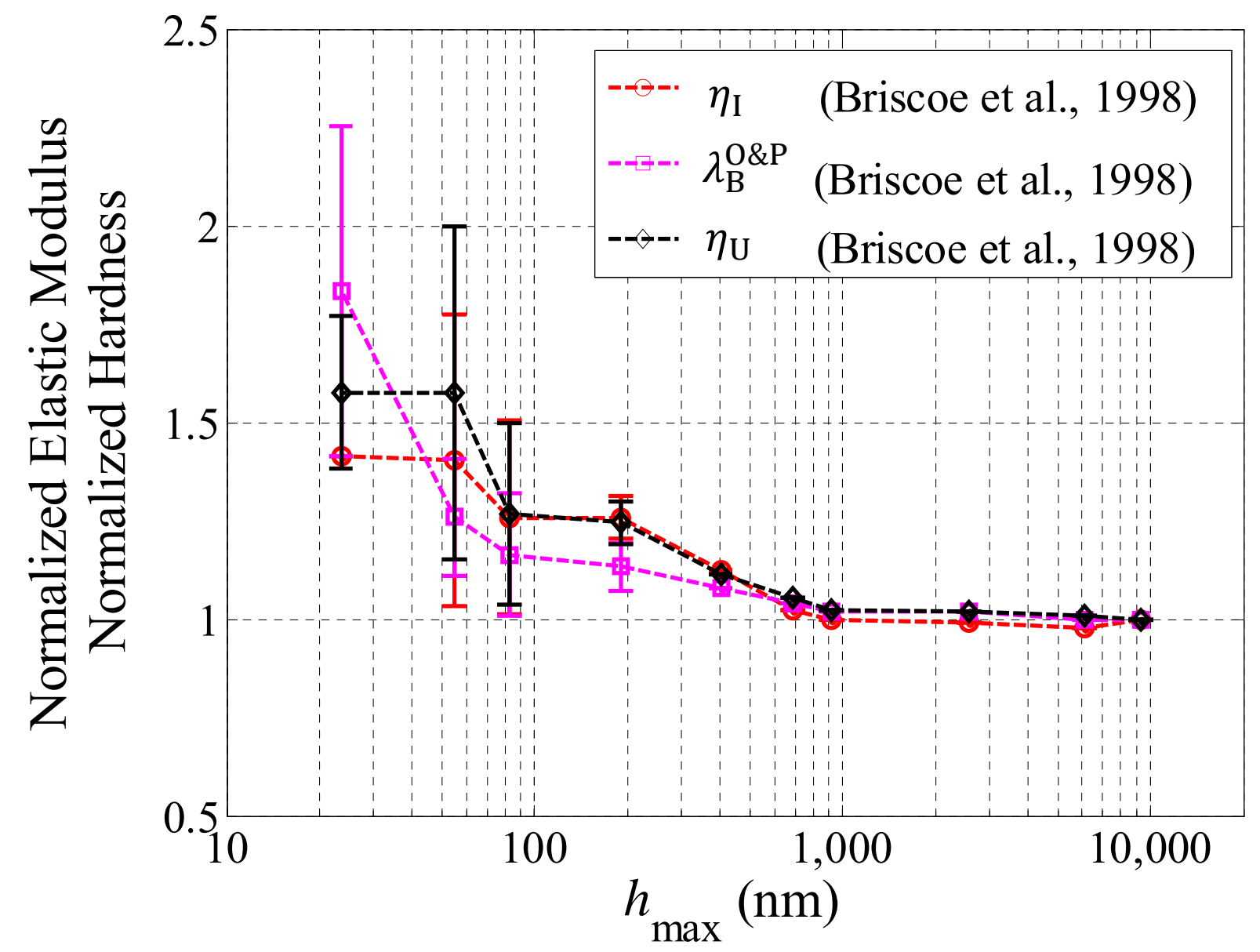

Poly methyl methacrylate (PMMA): Normalized values of indentation hardness, $\eta_{\mathrm{I}}=H_{\mathrm{I}} / H_{\mathrm{I}}^{(\mathrm{M})}$, elastic modulus according to Oliver \& Pharr (1992), $\lambda_{\mathrm{B}}^{\mathrm{O} \& \mathrm{P}}=E_{\mathrm{B}}^{\mathrm{O} \& \mathrm{P}} / E_{\mathrm{B}}^{\mathrm{O} \& \mathrm{P}(\mathrm{M})}$, and universal hardness, $\eta_{\mathrm{U}}=$ $H_{\mathrm{U}} / H_{\mathrm{U}}^{(\mathrm{M})}$, with $10 \mathrm{~s}$ loading, holding and unloading times (Briscoe et al., 1998) - superscript (M) indicates macroscopic values. 
Fig. 12 (a)

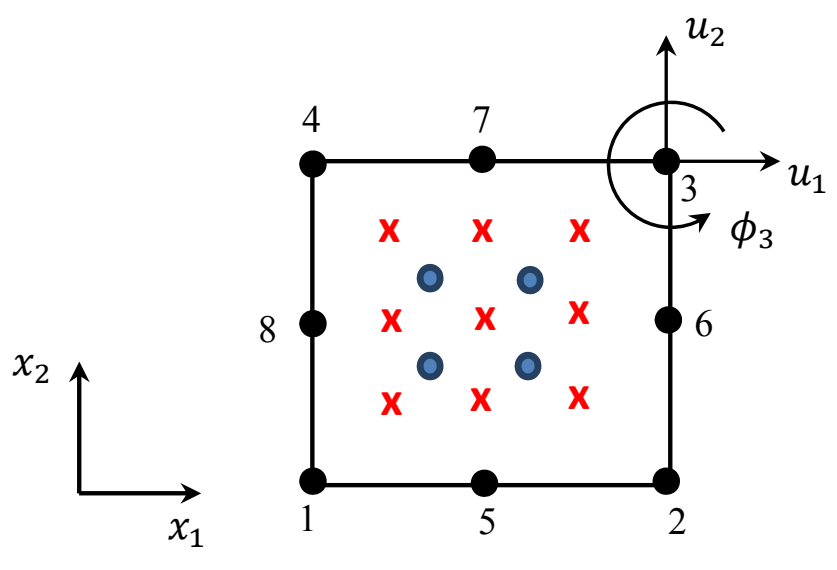

Degrees of freedom for 8-node element, full and reduced integration points are represented by $\mathrm{x}$ and 0 , respectively 
Fig. 12 (b)

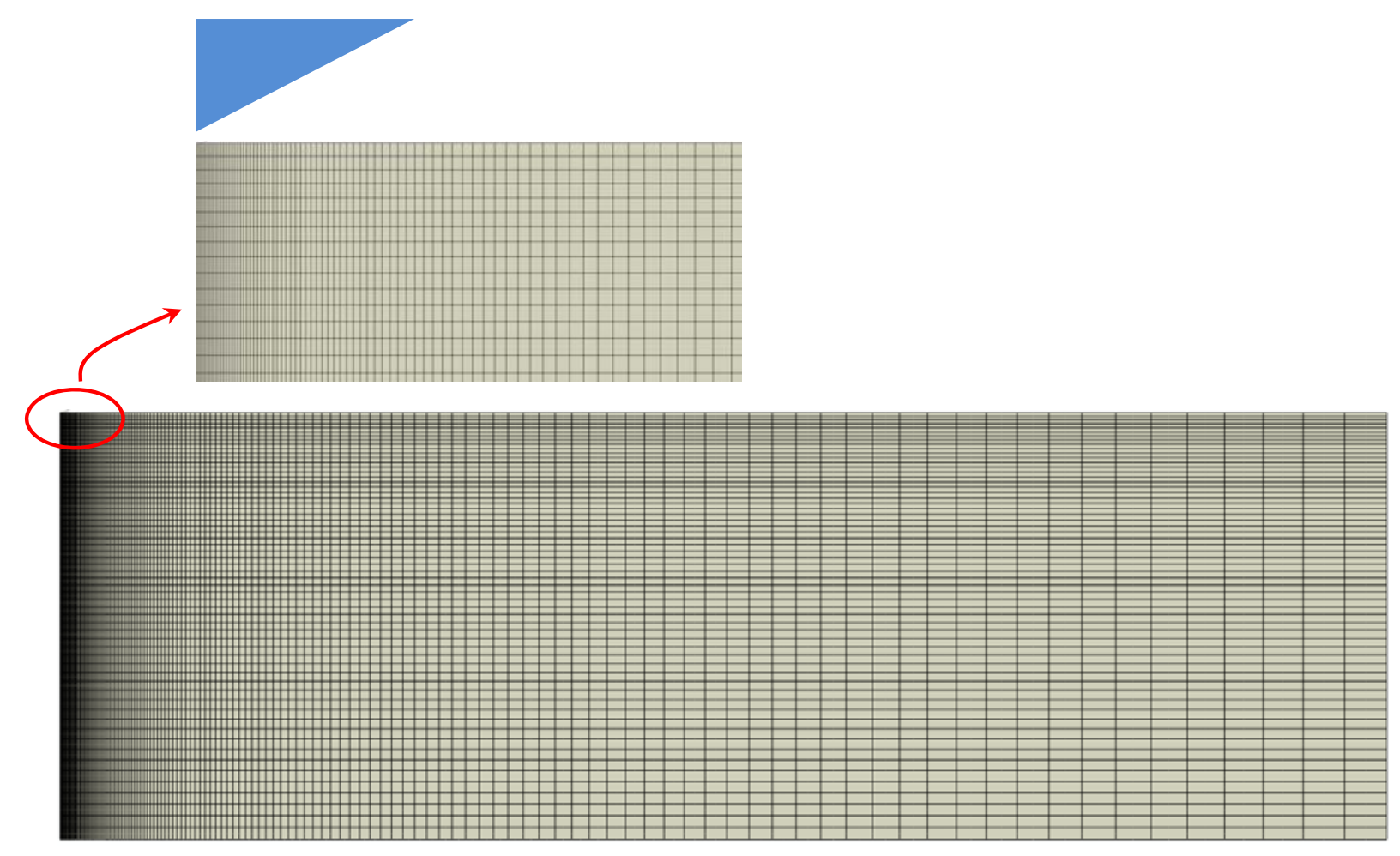

Finite element mesh for wedge indentation 
Fig. 13

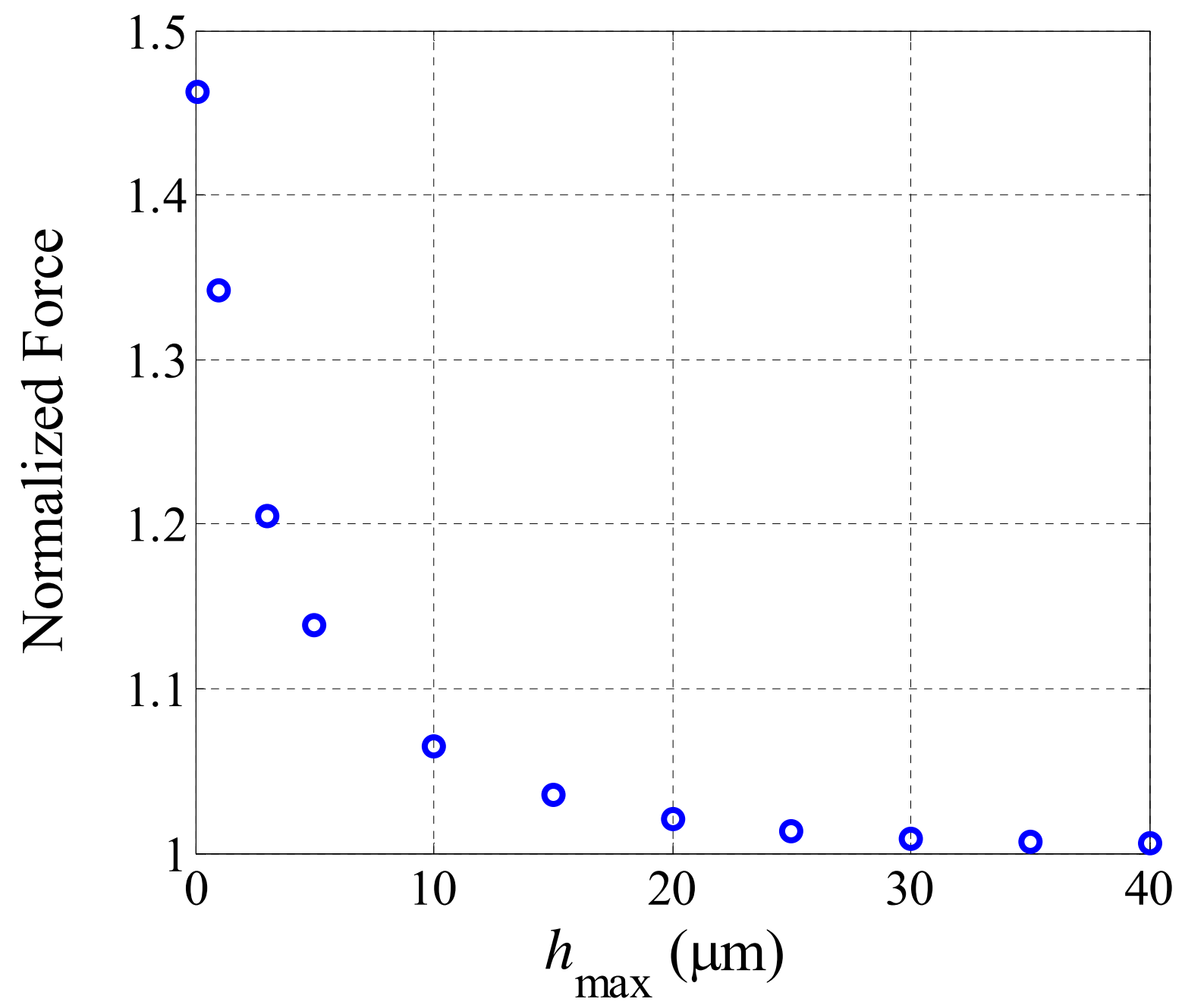

Normalized force versus $h_{\max }$ obtained from the wedge indentation simulations. Normalized force is calculated as the ratio of $F_{\mathrm{o}} / F_{*}$ where $F_{\mathrm{o}}$ is the reaction force when the effects of rotation gradients are taken into account (present formulation) and $F_{*}$ is the reaction force without the presence of rotation gradients (classical elasto-plasticity). 
Fig. 14

(a)

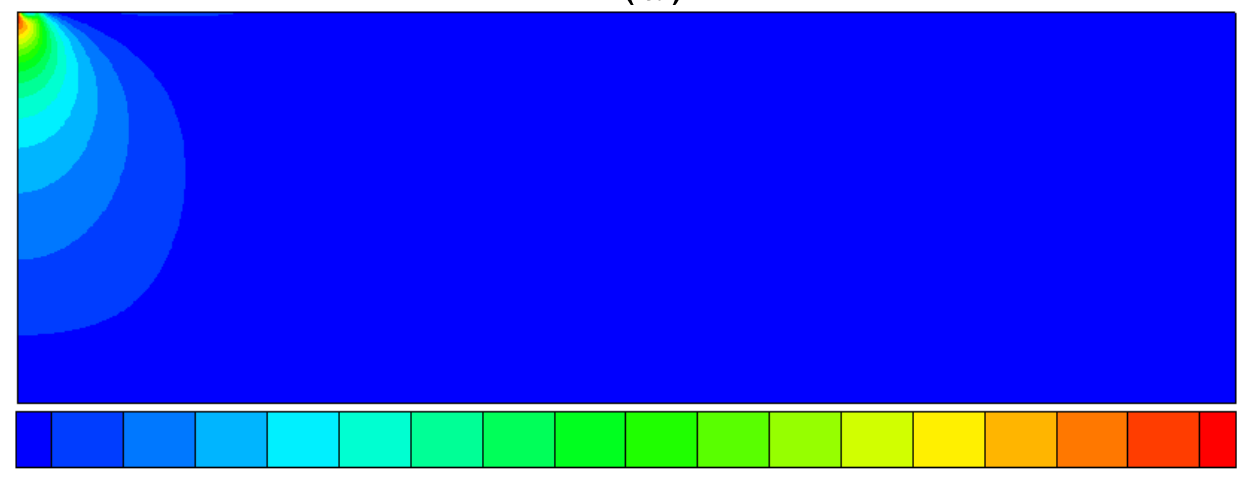

$\begin{array}{llllllllllllllllll}0.01 & 0.02 & 0.03 & 0.04 & 0.05 & 0.06 & 0.07 & 0.08 & 0.09 & 0.1 & 0.11 & 0.12 & 0.13 & 0.14 & 0.15 & 0.16 & 0.17\end{array}$

(b)

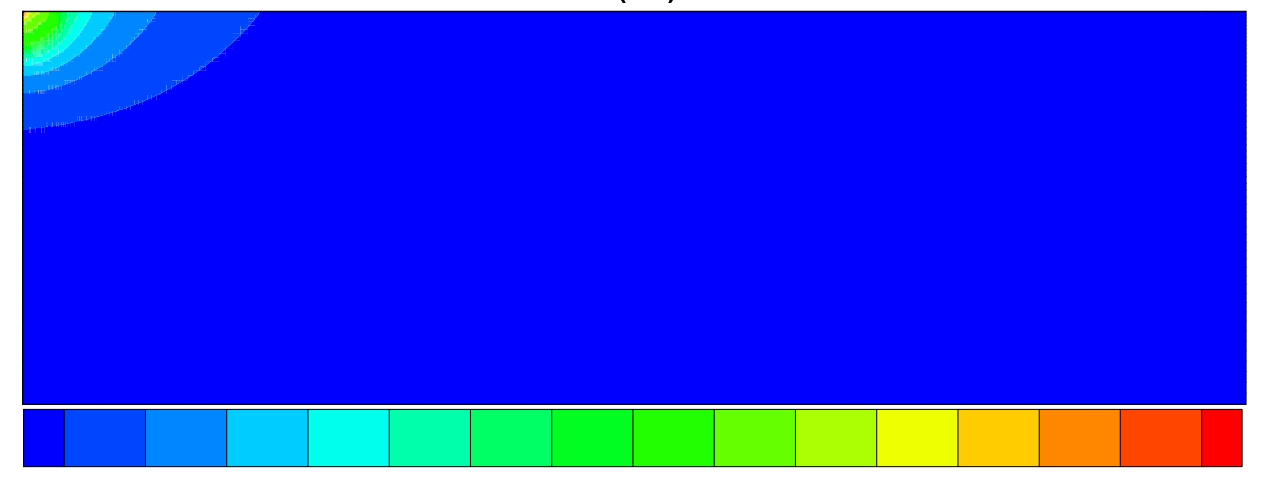

$\begin{array}{lllllllllllllll}0.02 & 0.04 & 0.06 & 0.08 & 0.1 & 0.12 & 0.14 & 0.16 & 0.18 & 0.2 & 0.22 & 0.24 & 0.26 & 0.28 & 0.3\end{array}$

Effective stress in GPa (a) without, and (b) with rotation gradients for $100 \mathrm{~nm}$ indentation of epoxy sample with length and thickness of 7 and $2.25 \mu \mathrm{m}$, respectively. 
Fig. 15

(a)

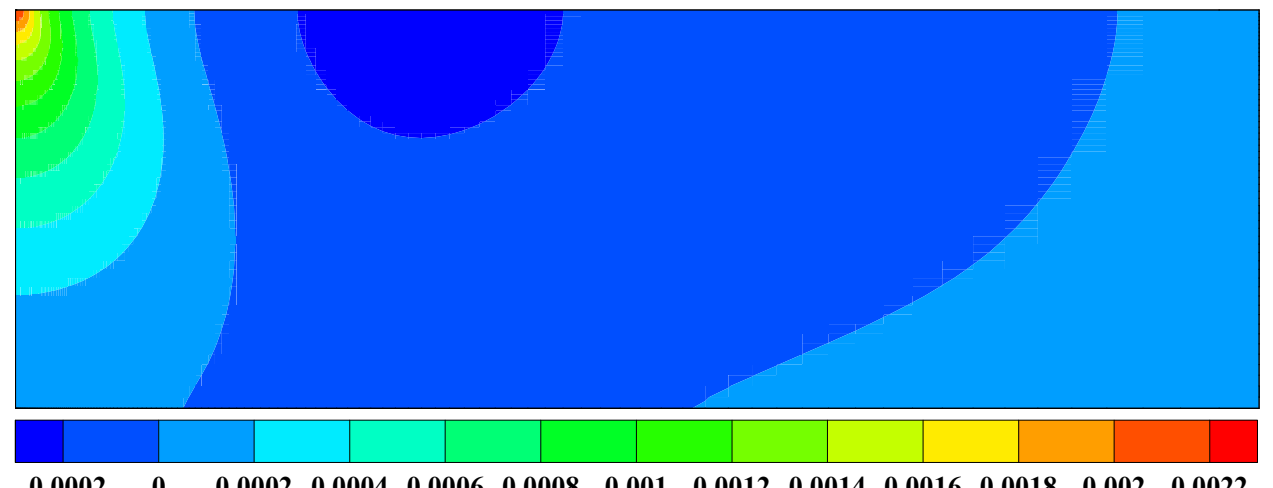

(b)

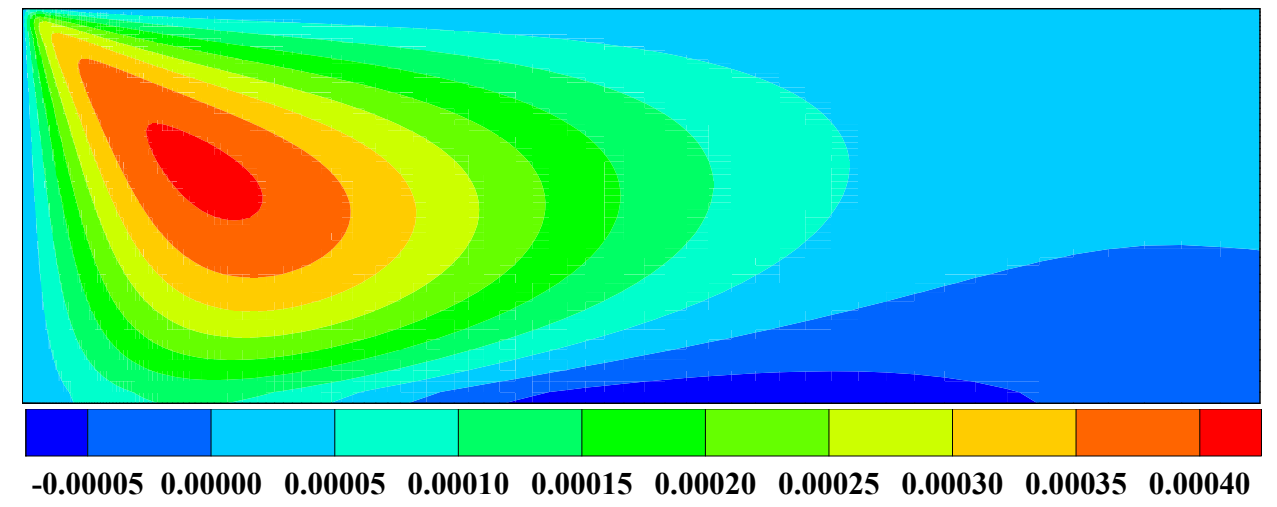

Rotation gradients (a) $\chi_{13}$, and (b) $\chi_{23}$ for $100 \mathrm{~nm}$ indentation of epoxy sample with length and thickness of 7 and $2.25 \mu \mathrm{m}$, respectively. 